\title{
Small airway-on-a-chip enables analysis of human lung inflammation and drug responses in vitro
}

\section{Citation}

Benam, Kambez H, Remi Villenave, Carolina Lucchesi, Antonio Varone, Cedric Hubeau, HyunHee Lee, Stephen E Alves, et al. 2015. "Small Airway-on-a-Chip Enables Analysis of Human Lung Inflammation and Drug Responses in Vitro." Nat Meth 13 (2) (December 21): 151-157. doi:10.1038/nmeth.3697.

\section{Published Version}

doi:10.1038/nmeth.3697

\section{Permanent link}

http://nrs.harvard.edu/urn-3:HUL.InstRepos:25623045

\section{Terms of Use}

This article was downloaded from Harvard University's DASH repository, and is made available under the terms and conditions applicable to Other Posted Material, as set forth at http:// nrs.harvard.edu/urn-3:HUL.InstRepos:dash.current.terms-of-use\#LAA

\section{Share Your Story}

The Harvard community has made this article openly available.

Please share how this access benefits you. Submit a story.

\section{Accessibility}


NATURE MTHODS - in press

Small airway-on-a-chip enables analysis of human lung inflammation and therapeutic responses in vitro

Kambez H. Benam ${ }^{1,8}$, Remi Villenave ${ }^{1,8}$, Carolina Lucchesi ${ }^{1,7}$, Antonio Varone ${ }^{1,7}$, Cedric Hubeau $^{2}$, Hyun-Hee Lee ${ }^{3}$, Stephen E. Alves ${ }^{3}$, Michael Salmon ${ }^{3}$, Thomas C. Ferrante ${ }^{1}$, James C. Weaver ${ }^{1,4}$, Geraldine A. Hamilton ${ }^{1,7}$, Anthony Bahinski ${ }^{1}$, and Donald E. Ingber ${ }^{1,4-6}$

${ }^{1}$ Wyss Institute for Biologically Inspired Engineering, Harvard University, Boston, Massachusetts, USA; ${ }^{2}$ Pfizer, Cambridge, Massachusetts, USA; ${ }^{3}$ Merck Research Laboratories, Boston, Massachusetts, USA; ${ }^{4}$ Harvard School of Engineering and Applied Sciences, Cambridge, Massachusetts, USA; ${ }^{5}$ Vascular Biology Program, Boston Children's Hospital, Boston, Massachusetts, USA; ${ }^{6}$ Harvard Medical School, Boston, Massachusetts, USA; ${ }^{7}$ Current address: Emulate Inc., Cambridge, Massachusetts, USA; ${ }^{8}$ These authors contributed equally to this work.

Corresponding Author: Donald E. Ingber, don.ingber@wyss.harvard.edu

Here we describe development of a human 'lung small airway-on-a-chip' containing a differentiated, mucociliary, bronchiolar epithelium and an underlying microvascular endothelium that experiences fluid flow, which enables analysis of organ-level lung pathophysiology in vitro. Exposure of the epithelium to IL-13 reconstitutes the goblet cell hyperplasia, cytokine hypersecretion and decreased ciliary function of asthmatics. Small airway chips lined by epithelial cells from chronic obstructive pulmonary disease patients recapitulate features of the disease including selective cytokine hypersecretion, increased neutrophil recruitment, and clinical exacerbations by exposure to viral and bacterial infections. Using this robust in vitro method for modeling human lung 
inflammatory disorders, it is possible to detect synergistic effects of lung

endothelium and epithelium on cytokine secretion, identify new biomarkers of

disease exacerbation, and measure therapeutic responses to anti-inflammatory

compounds that inhibit cytokine-induced recruitment of circulating neutrophils under flow.

Development of new therapeutics for chronic respiratory diseases, such as asthma and chronic obstructive pulmonary disease (COPD), which pose a huge public health burden ${ }^{1}$, have been hindered by the inability to study organ-level complexities of lung inflammation in vitro. While hospitalization and mortality due to these diseases are often the consequences of exacerbations triggered by pathogens ${ }^{2,3}$, there is currently no way to study these processes in human lung outside of the clinical setting. Animal models of asthma and COPD exist; however, their clinical relevance is questionable because the anatomy, immune system and inflammatory responses exhibited by animal lungs differ greatly from those in humans ${ }^{4-6}$. For example, mucin-producing cells, which are central to the development of asthma, are less frequent in the respiratory tree of mice and rats compared with humans ${ }^{6}$. Neutrophils that increase dramatically in the lungs of patients with COPD and severe asthma ${ }^{7-9}$ also comprise only $10-25 \%$ of circulating leukocytes in mice, whereas they represent $50-70 \%$ in humans ${ }^{5}$. Because many animal models fail to predict drug activities in humans, the pharmaceutical and biotechnology industries strive to reduce or replace animal models for drug testing whenever possible ${ }^{10}$. 
Airway inflammatory diseases have been modeled in vitro using cultures of primary or immortalized human epithelial cells, sometimes positioned at an air-liquid interface to induce epithelial differentiation ${ }^{11}$ or using co-cultures of airway epithelium and tissue-resident immune cells (e.g., macrophages or dendritic cells) ${ }^{12}$. However, lung inflammation is mediated by organ-level responses that involve complex tissuetissue interactions between the lung airway epithelium and underlying microvascular endothelium that modulate immune reactions to respiratory pathogens and allergens ${ }^{13-}$ ${ }^{15}$ and alter the vascular cell adhesion molecular machinery that recruits circulating immune cells, such as neutrophils. This is important because neutrophil accumulation in the lung is associated with enhanced severity of airflow limitation in COPD patients ${ }^{7}$ and it plays a critical role in severe asthma as well ${ }^{8}$. Unfortunately, it is not possible to study complex interactions among airway epithelium, endothelium and circulating neutrophils using existing in vitro lung models because most fail to recapitulate normal functional coupling between the epithelium and endothelium, and none enable analysis of recruitment of circulating immune cells under active fluid flow. This latter point is crucial because neutrophil adhesion to inflamed endothelium involves initial rolling along the luminal surface of endothelium mediated by E-selectin, which is then followed by firm adhesion to ICAM-1 (ref: 16) and this dynamic shear stress-dependent response cannot be studied in a physiologically relevant way using static cell cultures.

Advances in microsystems engineering have recently made it possible to create biomimetic microfluidic cell culture devices, known as 'organs-on-chips', that contain continuously perfused microchannels lined by living human cells that recapitulate the multicellular architectures, tissue-tissue interfaces, physicochemical microenvironments 
and vascular perfusion of the body ${ }^{17}$, which potentially offer new opportunities for disease modeling and drug efficacy assessment ${ }^{18}$. We previously used this approach to successfully reconstitute the alveolar-capillary interface of the human lung air sac and associated inflammatory responses in vitro ${ }^{19,20}$. Here, we adapted this approach to microengineer a human lung small airway-on-a-chip that supports full differentiation of a columnar, pseudostratified, mucociliary, bronchiolar epithelium composed of cells isolated from normal or COPD patients underlined by functional microvascular endothelium. We show that this model can be used to accurately reconstitute normal airway physiology as well as mimic complex human asthmatic and COPD inflammatory processes, including neutrophil recruitment and disease exacerbations in vitro. We also demonstrate that this in vitro tool provides a way to dissect contributions of individual tissue types (epithelium, endothelium and immune cells) to these organ-level responses, and to identify new anti-inflammatory therapies.

\section{RESULTS}

\section{On-chip reconstitution of a functional small airway}

To model the human small airway-on-a-chip, we used soft lithography to create a microfluidic device made of poly(dimethylsiloxane) (PDMS) containing an upper channel with a height and width (both $1 \mathrm{~mm}$ ) similar to the radius of a human bronchiole separated from a parallel lower microvascular channel $(0.2 \mathrm{~mm}$ high $\mathrm{Q} 1 \mathrm{~mm}$ wide) by a thin $(10 \mu \mathrm{m})$, porous $(0.4 \mu \mathrm{m})$, polyester membrane coated on both sides with type I collagen (Fig. 1a,b). We cultured primary human airway epithelial cells (hAECs) isolated from healthy donors or COPD patients on top of the membrane until confluent 
with medium flowing $\left(60 \mu \mathrm{L} \mathrm{h}^{-1}\right)$ in both channels. To trigger lung airway epithelial differentiation, the apical medium was removed after five days and air was introduced to create an air-liquid interface (ALI) while retinoic acid $\left(3 \mu \mathrm{g} \mathrm{ml}^{-1}\right)$ was added to the medium flowing in the lower channel to prevent the development of a squamous phenotype. Three to five weeks later, primary human lung microvascular endothelial cells were seeded on the opposite side of the porous membrane and cultured at the same flow rate until confluent to create a tissue-tissue interface (Fig. 1b,c).

Immunofluorescence confocal microscopic analysis revealed that these culture conditions resulted in the formation of a pseudo-stratified, mucociliary, airway epithelium and a planar microvascular endothelium on opposite sides of the same ECM-coated membrane (Fig. 1d-i and Supplementary Movie 1). The epithelial cells were linked by a continuous band of ZO1-containing tight junctions along the lateral borders of their apical surfaces (Fig. 1e), while endothelial cells were joined by adherens junctions containing PECAM-1 (Fig. 1f). This was accompanied by formation of a robust epithelial barrier that restricted the passage of fluorescent inulin $(4 \mathrm{kDa})$ and dextrans (10 and 70 $\mathrm{kDa}$ ) between the parallel channels when analyzed over $24 \mathrm{~h}$, mimicking the barrier function exhibited by living lung mucosa (Supplementary Fig.1a). Importantly, the epithelium formed from cells isolated from the small airway epithelium of lungs from both normal humans (Fig. 1g,i and Supplementary Movie 2) and COPD patients (Fig. 1h) contained many ciliated epithelial cells as well as mucus-producing Goblet cells (Fig. 1g,h), Club cells (Supplementary Fig. 1b) and Basal cells that appeared in strikingly similar proportions to those found in normal human lung (Table 1). Electron microscopic analysis of the normal lung small airway-on-a-chip confirmed that the cilia that appeared 
on the apical surface of the polarized epithelium (Fig. 1i) also exhibited the same structure $(9+2$ microtubule organization) and length $(\sim 6 \mu \mathrm{m})$ (Supplementary Fig. 1c) as healthy cilia found in living human lung in vivo ${ }^{21,22}$. Importantly, high-speed microscopic imaging confirmed that these cilia beat actively at a frequency of $9-20 \mathrm{~Hz}$ (Fig. 1j and Supplementary Movie 3), which again is nearly the same as that observed in human airways ${ }^{21,23}$ (Table 1). Moreover, when mucociliary transport was measured by introducing fluorescent polystyrene microbeads into the top channel, rapid coordinated movement of the beads were observed along the surface of the epithelium (Fig. 1k and Supplementary Movie 4) as a result of active synchronized cilia beating; again, the particle velocity measured $(50-100 \mu \mathrm{m} / \mathrm{sec})$ was nearly identical to that observed in the normal human lung airway (Table 1). Thus, the human lung small airway-on-a-chip effectively recapitulates many of the structures and functions of the normal lung bronchiole and sustains them for weeks in vitro.

\section{Modeling asthma and lung inflammation on-chip}

IL-13 plays a pivotal role in asthma as it is necessary and sufficient to induce all features of allergic asthma in animal models of the disease ${ }^{24}$. IL-13 has a direct effect on the human airway epithelium ${ }^{25}$ and participates in airway inflammation, goblet cell hyperplasia and mucus hypersecretion as well as sub-epithelial fibrosis and airway hyper-responsiveness ${ }^{24}$. Importantly, when the small airway-on-a-chip was treated with IL-13 (100 $\left.\mathrm{ng} \mathrm{ml}^{-1}\right)$ for 8 days, there were significant increases in Goblet cell number (Fig. 2a, b) in line with other in vitro models ${ }^{26}$, higher production and secretion of the inflammatory cytokines, G-CSF and GM-CSF, into the vascular channel as measured in 
the fluid effluent at day 2 and 8 respectively (Fig. 2c), and a reduction in cilia beating frequency (Fig. 2d) similar to that observed in the airway mucosa of asthma patients ${ }^{27-29}$.

As mucosal inflammation and its exacerbation by viral infections are major components of both asthma and COPD, and it has been challenging to recapitulate these clinically relevant responses in vitro, we analyzed the effects of exposing the airway epithelium to the viral mimic polyinosinic-polycytidylic acid (poly I:C) $\left(10 \mu \mathrm{g} \mathrm{ml}^{-1}\right)$. Poly I:C is a synthetic analogue of the double-stranded RNA that is produced in infected cells during viral replication by respiratory viruses ${ }^{30}$. Stimulation with poly I:C for $3 \mathrm{~h}$ triggered a pro-inflammatory response in the chip similar to that which occurs in patients with acute severe asthma exacerbations ${ }^{8}$, including large increases in basal secretion of RANTES, IL-6 and IP-10 (Fig. 2e), which are also cytokines (or chemokines) that accumulate in lungs of human patients infected with respiratory viruses ${ }^{15}$. In addition, poly I:C induced upregulation of E-selectin and VCAM-1 expression in the underlying endothelium (Supplementary Fig. 2a), which are crucial for the initial adhesion and rolling of neutrophils under flow within microvessels in inflamed living tissues ${ }^{31}$. In fact, this resulted in enhanced adhesion, rolling and total recruitment of circulating human neutrophils in the small airway chip when primary human neutrophils were flowed through the lower channel under physiological flow and shear stress $\left(1 \mathrm{dyne} \mathrm{cm}^{-2}\right)$ (Fig.

\section{2f,g and Supplementary Movie 5).}

Because of the synthetic nature of our engineered lung airway model, we were able to analyze the independent and collective contributions of the lung epithelium and endothelium to this inflammatory response, which is not possible in human subjects. While previous reports have investigated airway epithelium homeostasis using co- 
cultures of endothelium and lung basal cells ${ }^{32,33}$, inflammatory response using a fully differentiated human small airway epithelium has not been previously demonstrated in vitro. Interestingly, treatment of the differentiated epithelium with the same dose of poly $\mathrm{I}: \mathrm{C}$ in the presence of an underlying endothelium resulted in a 3- to 6-fold increase in the levels of secreted RANTES, IL-6 and IP-10 relative to levels when the endothelium was absent (Fig. 2e), and this effect appeared to be specific for these cytokines in that removal of the endothelium had no effect on the secretion of GRO $\alpha$ or IL-8

(Supplementary Fig. 2b). Importantly, we showed that this effect was synergistic as stimulated endothelial cells alone secreted similar or lower level of cytokines than the isolated epithelium (Fig. 2e). Thus, these findings reveal an intrinsic cross-talk between human epithelium and endothelium during poly I:C-induced inflammation and corroborate past animal studies, which suggest that the pulmonary endothelium contributes significantly to viral-induced inflammation by acting as a central regulator of the cytokine storm ${ }^{15}$. Also, when we compared responses to poly I:C stimulation in bronchiolar versus bronchial epithelial cells, we found that they both displayed nearly identical secretion of inflammatory cytokines (Supplementary Fig. 2c).

\section{Modeling COPD exacerbation on-chip}

As pathogenic infections are a major cause of COPD exacerbations in patients, we next explored whether the lung small airway-on-a-chip also could be used to model these inflammatory responses in vitro. We stimulated small airway chips lined by normal or COPD epithelial cells with the viral mimic poly I:C or lipopolysaccharide endotoxin (LPS), which is a bacterial wall-derived component that stimulates cytokine production and has been widely used to simulate bacterial infections in vitro ${ }^{34}$. We analyzed 
secretion of the cytokine M-CSF because it promotes differentiation and survival of macrophages, and production of IL-8 because it attracts neutrophils, as these are two major immune cells types that accumulate in the airway tissue of COPD patients ${ }^{35,36}$. Interestingly, stimulation with LPS and poly I:C respectively upregulated secretion of IL8 and M-CSF in the COPD chips, but these infection mimics did not produce any significant change in chips lined by healthy airway epithelial cells (Fig. 3). These results are consistent with past findings that showed bronchial epithelial spheroids from COPD patients exhibit higher LPS-induced IL-8 release compared with healthy controls ${ }^{37}$, and the significantly enhanced levels of M-CSF we detected might partly explain the elevated pulmonary accumulation of macrophages that is observed in lungs of COPD patients $^{9}$. In contrast, stimulation with poly I:C produced large increases in secretion of the cytokines IP-10 and RANTES in both healthy and COPD chips (Fig. 3). These results are consistent with the finding that serum IP-10 is an excellent clinical marker for acute viral exacerbations in COPD patients ${ }^{2}$. Importantly, our finding that M-CSF levels specifically increase only in poly I:C treated small airway chips (Fig. 3) suggests that MCSF could represent a new biomarker for COPD exacerbations induced by respiratory viruses.

\section{Evaluation of therapeutic responses in small airway chips}

Having established these microfluidic models of human asthmatic and COPD airways, we then asked whether this new in vitro method could be useful for drug discovery. As IL-13 signals through JAK-Stat pathway, we investigated whether Tofacitinib, a potent inhibitor of JAK1, 2 and 3 used for patients with rheumatoid arthritis $^{38}$, could reverse the IL-13-induced phenotype. When we exposed normal airway 
epithelium on-chip to IL-13 to produce asthmatic changes in the epithelium, and then treated with high doses of Tofacitinib ( 1 and $10 \mu \mathrm{M}$ ) to inhibit JAK signaling, we found that it suppressed goblet cell hyperplasia, decreased secretion of G-CSF and GM-CSF, and restored normal cilia beating frequency (Fig. 4a,b). In contrast, when similar chips exposed to IL-13 were treated with the corticosteroid, dexamethasone, it was found to be ineffective, which is consistent with the clinical finding that although moderate to severe asthma patients receive dexamethasone inhalation therapy, they often fail to respond to this treatment ${ }^{39}$. These results also validate recent reports suggesting that immunosuppressant JAK inhibitors may represent potential new therapeutics for allergic airway diseases ${ }^{40}$.

To test the breadth and robustness of our platform, we also explored whether we can use the human small airway-on-a-chip model of COPD exacerbation by microbial infection to identify pharmacological agents that can suppress associated inflammation, specifically by inhibiting recruitment of neutrophils circulating under flow. When we treated COPD epithelial cells that were stimulated with poly I:C on-chip with the glucocorticoid drug, budesonide, there was no significant change in neutrophil adhesion (Fig. 4c,d), which again is consistent with its noted lack of activity in many COPD patients ${ }^{41}$. We then tested a new experimental anti-inflammatory drug, 2-methoxy-N-(3methyl-2-oxo-1,2-dihydroquinolin-6-yl) benzenesulfonamide ${ }^{42}$, which is a Bromodomain Containing Protein 4 (BRD4) inhibitor of NFKB signaling, because it belongs to an emerging class of new anti-inflammatory drugs that were recently shown to alleviate lung inflammation in an LPS-challenged mouse model ${ }^{43}$. Interestingly, when the BRD4 inhibitor was added to the inflamed human small airway chip, it suppressed neutrophil 
adhesion under flow by almost $75 \%$ in the chip (Fig. 4c,d). However, when we tested these same drugs on similarly differentiated airway epithelium maintained with an underlying endothelium in static Transwell cultures, the inhibitory effect of the BRD4 inhibitor on recruitment of added neutrophils was reduced by one third, even though similar results were obtained with budesonide (Fig. 4d). This differential response to the BRD4 inhibitor under static versus dynamic flow conditions suggests that its mechanism of action might involve preferential inhibition of early adhesion and rolling responses mediated that are required for initiation of the neutrophil recruitment cascade because they only occur under dynamic flow conditions ${ }^{44}$. In fact, our studies revealed that treatment of the inflamed endothelial cells with the BRD4 inhibitor resulted in more than a 50 -fold reduction in the expression levels of E-selectin and VCAM-1, as well as a $50 \%$ reduction in ICAM-1, whereas budesonide was unable to significantly reduce expression of either these vascular cell adhesion molecules (Fig. 4e).

Consistent with its ability to suppress neutrophil adhesion, this BRD4 inhibitor also proved greatly superior to the corticosteroid in down-regulating expression of the IL-8, MCP-1, GROa, and IL-6 genes in human lung microvascular endothelial cells (Supplementary Fig. 3), as well as suppressing secretion of these cytokines into the lower microvascular channel (Fig. 5). These are important findings because these cytokines are key chemoattractants for neutrophils in vivo. This BRD4 inhibitor also significantly suppressed production of GM-CSF (Fig. 5), which is a neutrophil chemokine that enhances functionality of neutrophils ${ }^{45}$, in addition to supporting bone marrow cell proliferation and survival of granulocytic precursors. Interestingly, while we found that budesonide slightly lowered expression of selected cytokine genes 
(Supplementary Fig. 3), it failed to significantly suppress cytokine secretion (Fig. 5), which is the most critical determinant of the inflammatory response, again mimicking results that have been observed in animal models of COPD and lung inflammation ${ }^{43,46}$. Thus, reconstitution of the COPD inflammatory phenotype on-chip not only enabled testing for efficacy of new experimental therapeutics, it also allowed dissection of the mechanism of drug action at the molecular level in a human organ context in vitro.

\section{DISCUSSION}

In this article, we described a method to engineer a human lung small airway-ona-chip containing living, fully differentiated, pseudo-stratified, mucociliary, human bronchiolar epithelium as well as an underlying human pulmonary microvascular endothelium and immune cells that circulate through the artificial microvessel under flow. We also demonstrated how this small airway chip can be used to model and study human pulmonary inflammatory diseases, recapitulating many of the features asthma and COPD seen in human patients, as well as to assess potential therapeutic responses. While past attempts were made to culture human airway epithelium in microfluidic devices along with endothelium as well as fibroblasts ${ }^{47}$, this level of robustness and high fidelity of mimicking the structure and functions of human lung airway, including modeling of pulmonary diseases and recapitulation of lung inflammatory responses, have never been achieved previously.

Because the organ-on-chip approach essentially represents 'synthetic biology' at the organ level, we also were able to independently control and vary virtually all system parameters, including the presence or absence of different cell types, air and vascular 
flow conditions, and soluble factors, while simultaneously analyzing human organ-level responses in real-time with molecular scale resolution. Using this approach, we gained new insight into how airway epithelium and microvascular endothelium interplay to control cytokine production and regulate immune cell recruitment, identified a potential new clinical biomarker, and demonstrated the feasibility of using this method to screen for new anti-inflammatory therapeutics. This microengineered lung small airway-on-achip model that faithfully recapitulates in vivo organ-level responses to therapy therefore offers a powerful complement to animal models for both analyzing human pathophysiology and carrying out preclinical drug evaluation (see Supplementary Discussion for more details).

\section{ACKNOWLEDGMENTS}

Funding was provided by Pfizer, Merck, Wyss Institute for Biologically Inspired Engineering at Harvard University, and Defense Advanced Research Projects Agency (DARPA) under Cooperative Agreement Number W911NF-12-2-0036. We thank K. Karalis for helpful discussions, and B. Hassell and M. Mazur for their technical assistance.

\section{AUTHOR CONTRIBUTIONS}

K.H.B., R.V., C.H., H.H.L., S.E.A., M.S. and D.E.I. designed the research; K.H.B. and R.V. developed the basic small airway chip model; R.V. and A.V. conducted the asthma work; K.H.B. and C.L. conducted the COPD studies; K.H.B. optimized and performed leukocyte recruitment studies; J.C.W. performed scanning electron 
microscopy imaging; T.C.F. helped with confocal microscopy imaging; K.H.B., and R.V. prepared the manuscript; G.A.H. and A.B. commented on the manuscript; D.E.I. critically revised the manuscript.

\section{COMPETING OF COMPETING FINANCIAL INTERESTS}

D.E. Ingber and G.A. Hamilton are founders and hold equity in Emulate, Inc., and Ingber chairs its scientific advisory board. 


\section{REFERENCES}

1. Top 10 Causes of Death. World Health Organization. http://www.who.int/mediacentre/factsheets/fs310/en/.

2. Bafadhel, M. et al. Acute exacerbations of chronic obstructive pulmonary disease: identification of biologic clusters and their biomarkers. American journal of respiratory and critical care medicine 184, 662-671 (2011).

3. Jackson, D.J., Sykes, A., Mallia, P. \& Johnston, S.L. Asthma exacerbations: origin, effect, and prevention. The Journal of allergy and clinical immunology 128 , 1165-1174 (2011).

4. Hyde, D.M., Hamid, Q. \& Irvin, C.G. Anatomy, pathology, and physiology of the tracheobronchial tree: emphasis on the distal airways. The Journal of allergy and clinical immunology 124, S72-77 (2009).

5. Kolaczkowska, E. \& Kubes, P. Neutrophil recruitment and function in health and inflammation. Nature reviews. Immunology 13, 159-175 (2013).

6. Wright, J.L., Cosio, M. \& Churg, A. Animal models of chronic obstructive pulmonary disease. American journal of physiology. Lung cellular and molecular physiology 295, L1-15 (2008).

7. Di Stefano, A. et al. Severity of airflow limitation is associated with severity of airway inflammation in smokers. American journal of respiratory and critical care medicine 158, 1277-1285 (1998).

8. Fahy, J.V. Eosinophilic and neutrophilic inflammation in asthma: insights from clinical studies. Proceedings of the American Thoracic Society 6, 256-259 (2009). 
9. Hogg, J.C. et al. The nature of small-airway obstruction in chronic obstructive pulmonary disease. The New England journal of medicine 350, 2645-2653 (2004).

10. Bueters, T., Ploeger, B.A. \& Visser, S.A. The virtue of translational PKPD modeling in drug discovery: selecting the right clinical candidate while sparing animal lives. Drug discovery today 18, 853-862 (2013).

11. Fulcher, M.L., Gabriel, S., Burns, K.A., Yankaskas, J.R. \& Randell, S.H. Welldifferentiated human airway epithelial cell cultures. Methods in molecular medicine 107, 183-206 (2005).

12. Ugonna, K., Bingle, C.D., Plant, K., Wilson, K. \& Everard, M.L. Macrophages are required for dendritic cell uptake of respiratory syncytial virus from an infected epithelium. PloS one 9, e91855 (2014).

13. Didierlaurent, A. et al. Sustained desensitization to bacterial Toll-like receptor ligands after resolution of respiratory influenza infection. The Journal of experimental medicine 205, 323-329 (2008).

14. Hammad, H. et al. House dust mite allergen induces asthma via Toll-like receptor 4 triggering of airway structural cells. Nature medicine 15, 410-416 (2009).

15. Teijaro, J.R. et al. Endothelial cells are central orchestrators of cytokine amplification during influenza virus infection. Cell 146, 980-991 (2011).

16. Langer, H.F. \& Chavakis, T. Leukocyte-endothelial interactions in inflammation. Journal of cellular and molecular medicine 13, 1211-1220 (2009).

17. Bhatia, S.N. \& Ingber, D.E. Microfluidic organs-on-chips. Nature biotechnology 32, 760-772 (2014). 
18. Esch, E.W., Bahinski, A. \& Huh, D. Organs-on-chips at the frontiers of drug discovery. Nature reviews. Drug discovery 14, 248-260 (2015).

19. Huh, D. et al. A human disease model of drug toxicity-induced pulmonary edema in a lung-on-a-chip microdevice. Science translational medicine 4, 159 ra147 (2012).

20. Huh, D. et al. Reconstituting organ-level lung functions on a chip. Science 328, 1662-1668 (2010).

21. Wanner, A., Salathe, M. \& O'Riordan, T.G. Mucociliary clearance in the airways. American journal of respiratory and critical care medicine 154, 1868-1902 (1996).

22. Fliegauf, M., Benzing, T. \& Omran, H. When cilia go bad: cilia defects and ciliopathies. Nature reviews. Molecular cell biology 8, 880-893 (2007).

23. Livraghi, A. \& Randell, S.H. Cystic fibrosis and other respiratory diseases of impaired mucus clearance. Toxicologic pathology 35, 116-129 (2007).

24. Wills-Karp, M. Interleukin-13 in asthma pathogenesis. Immunological reviews 202, 175-190 (2004).

25. Kuperman, D.A. et al. Direct effects of interleukin-13 on epithelial cells cause airway hyperreactivity and mucus overproduction in asthma. Nature medicine $\mathbf{8}$, 885-889 (2002).

26. Danahay, $\mathrm{H}$. et al. Notch2 is required for inflammatory cytokine-driven goblet cell metaplasia in the lung. Cell reports 10, 239-252 (2015).

27. Sousa, A.R., Poston, R.N., Lane, S.J., Nakhosteen, J.A. \& Lee, T.H. Detection of GM-CSF in asthmatic bronchial epithelium and decrease by inhaled 
corticosteroids. The American review of respiratory disease 147, 1557-1561 (1993).

28. Ordonez, C.L. et al. Mild and moderate asthma is associated with airway goblet cell hyperplasia and abnormalities in mucin gene expression. American journal of respiratory and critical care medicine 163, 517-523 (2001).

29. Thomas, B. et al. Ciliary dysfunction and ultrastructural abnormalities are features of severe asthma. The Journal of allergy and clinical immunology 126, 722-729 e722 (2010).

30. Hewson, C.A., Jardine, A., Edwards, M.R., Laza-Stanca, V. \& Johnston, S.L. Toll-like receptor 3 is induced by and mediates antiviral activity against rhinovirus infection of human bronchial epithelial cells. Journal of virology $\mathbf{7 9}, 12273-12279$ (2005).

31. Lawrence, M.B. \& Springer, T.A. Neutrophils roll on E-selectin. J Immunol 151, 6338-6346 (1993).

32. Curradi, G. et al. Airway basal cell vascular endothelial growth factor-mediated cross-talk regulates endothelial cell-dependent growth support of human airway basal cells. Cellular and molecular life sciences : CMLS 69, 2217-2231 (2012).

33. Ding, B.S., Gomi, K., Rafii, S., Crystal, R.G. \& Walters, M.S. Endothelial MMP14 is required for endothelial-dependent growth support of human airway basal cells. Journal of cell science 128, 2983-2988 (2015).

34. Mayer, A.K. et al. Differential recognition of TLR-dependent microbial ligands in human bronchial epithelial cells. J Immunol 178, 3134-3142 (2007).

35. O'Shaughnessy, T.C., Ansari, T.W., Barnes, N.C. \& Jeffery, P.K. Inflammation in bronchial biopsies of subjects with chronic bronchitis: inverse relationship of 
CD8+ T lymphocytes with FEV1. American journal of respiratory and critical care medicine 155, 852-857 (1997).

36. Papi, A. et al. Infections and airway inflammation in chronic obstructive pulmonary disease severe exacerbations. American journal of respiratory and critical care medicine 173, 1114-1121 (2006).

37. Deslee, G. et al. Bronchial epithelial spheroids: an alternative culture model to investigate epithelium inflammation-mediated COPD. Respiratory research 8, 86 (2007).

38. Fleischmann, R. et al. Placebo-controlled trial of tofacitinib monotherapy in rheumatoid arthritis. The New England journal of medicine 367, 495-507 (2012).

39. Bush, A. et al. Severe childhood asthma: a common international approach? Lancet 372, 1019-1021 (2008).

40. Kudlacz, E., Conklyn, M., Andresen, C., Whitney-Pickett, C. \& Changelian, P. The JAK-3 inhibitor CP-690550 is a potent anti-inflammatory agent in a murine model of pulmonary eosinophilia. European journal of pharmacology 582, 154161 (2008).

41. Ammit, A.J. Glucocorticoid insensitivity as a source of drug targets for respiratory disease. Current opinion in pharmacology 13, 370-376 (2013).

42. Wu, J. et al. Design and chemoproteomic functional characterization of a chemical probe targeted to bromodomains of BET family proteins. Med. Chem. Commun. 5, 1871-1878 (2014).

43. Belkina, A.C., Nikolajczyk, B.S. \& Denis, G.V. BET protein function is required for inflammation: Brd2 genetic disruption and BET inhibitor JQ1 impair mouse macrophage inflammatory responses. J Immunol 190, 3670-3678 (2013). 
44. Abbassi, O., Kishimoto, T.K., Mclntire, L.V., Anderson, D.C. \& Smith, C.W. Eselectin supports neutrophil rolling in vitro under conditions of flow. The Journal of clinical investigation 92, 2719-2730 (1993).

45. Gomez-Cambronero, J., Horn, J., Paul, C.C. \& Baumann, M.A. Granulocytemacrophage colony-stimulating factor is a chemoattractant cytokine for human neutrophils: involvement of the ribosomal p70 S6 kinase signaling pathway. $J$ Immunol 171, 6846-6855 (2003).

46. Hubeau, C., Kubera, J.E., Masek-Hammerman, K. \& Williams, C.M. Interleukin-6 neutralization alleviates pulmonary inflammation in mice exposed to cigarette smoke and poly(l:C). Clin Sci (Lond) 125, 483-493 (2013).

47. Sellgren, K.L., Butala, E.J., Gilmour, B.P., Randell, S.H. \& Grego, S. A biomimetic multicellular model of the airways using primary human cells. Lab on a chip 14, 3349-3358 (2014).

48. Boers, J.E., Ambergen, A.W. \& Thunnissen, F.B. Number and proliferation of clara cells in normal human airway epithelium. American journal of respiratory and critical care medicine 159, 1585-1591 (1999).

49. Mercer, R.R., Russell, M.L., Roggli, V.L. \& Crapo, J.D. Cell number and distribution in human and rat airways. American journal of respiratory cell and molecular biology 10, 613-624 (1994). 


\section{FIGURE LEGENDS}

Figure 1. The human small airway-on-a-chip. (a) Photograph of the microfluidic small airway-on-a-chip device (airway channel is filled with blue dye; microvascular channel is filled in yellow; bar, $1 \mathrm{~cm}$ ). (b) Schematic diagram of a cross section through the small airway-on-a-chip shown in a, visualizing its two hollow linear channels separated by a thin, porous, collagen-coated membrane which supports growth and differentiation of human primary airway epithelial cells on its upper surface and human pulmonary microvascular endothelial cells on its lower surface. (c) Diagram of the tissue-tissue interface that forms on-chip, with a differentiated airway epithelium cultured on top of the porous collagen-coated membrane at an air-liquid interface in the upper channel, and the endothelium below with medium flowing beneath it that feeds both tissue layers. (d-h) Confocal immunofluorescence views of cells grown for 3 to 5 weeks at air liquid interface in the small airway chip (bar, $20 \mu \mathrm{m}$ ). (d) A 3D reconstruction showing fully differentiated, pseudostratified, airway epithelium formed on-chip by cultured hAECs (green, F-actin) underlined by human pulmonary microvascular endothelial cells (red, F-actin) on the opposite side of the membrane (blue, DAPI-stained nuclei). (e) The differentiated human airway epithelium exhibits continuous tight junctional connections on-chip, as evidenced by ZO1 staining (red). (f) The human (lung blood microvascular) endothelial monolayer formed on chip also contains continuous adherens junctions between adjacent cells, as indicated by PECAM-1 staining (green). Well-differentiated human airway epithelium formed on-chip using hAECs derived from healthy $(\mathbf{g})$ versus COPD donors $(\mathbf{h})$ demonstrating presence of high numbers of ciliated cells labeled for $\beta$-tubulin IV (green) and goblet cells stained 
with anti-MUC5AC antibody (magenta). (i) Scanning electron micrograph of cilia on the apical surface of the differentiated airway epithelium formed on-chip (cilia were colored in blue and non-ciliated cells in brown; bar, $10 \mu \mathrm{m}$ ). (j) Sequential frames of a movie of the apical surface of a differentiated epithelium recorded over 100 milliseconds demonstrating cilia beating at a frequency of $\sim 10 \mathrm{~Hz}$. A single cilium is highlighted in white; time stamps and arrows indicate the duration and direction of one forward and return stroke (full movie is shown in Supplementary Movie 4; bar, $5 \mu \mathrm{m}$ ). (k) Synchronized cilia beating visualized by adding $1 \mu \mathrm{m}$ fluorescent beads to the apical side of an empty chip (left) compared to its addition to a small airway chip device with a fully differentiated, living airway epithelium (right; image was recorded over $1 \mathrm{sec}$; bar, $50 \mu \mathrm{m})$. All images are representative of 3-6 independent experiments performed in 36 different donors.

Figure 2. Modeling asthma and lung inflammation on-chip.

(a) Immunofluorescence micrographic views of the differentiated airway epithelium cultured on-chip for 4 to 6 weeks at air liquid interface in the absence or presence of IL13 showing the epithelium stained for the goblet cell marker, MUC5AC (green) and DAPI (blue; bar, $50 \mu \mathrm{m}$; representative of 3 independent experiments performed in 3 different donors), (b) graphic depictions of total culture area covered by goblet cells, (c) production of the cytokines GM-CSF and G-CSF (c) and cilia beating frequency (d) under these conditions (unpaired Student's $t$-test; * $P<0.05,{ }^{* *} P<0.01$, ${ }^{* * *} P<0.001$; data represent mean \pm s.e.m (compared to unstimulated) from 3 healthy donors, with 1 2 biological replicates per donor; for goblet cell analysis $3-5$ representative areas per condition were used for quantification; $n=3-8$ ). (e) Production of the cytokines 
RANTES, IL-6 and IP-10 by the small airway-on-a-chip with (+) or without (-) a differentiated bronchiolar epithelium, an umbilical vein endothelium, or both, in the presence or absence of poly I:C $\left(10 \mu \mathrm{g} \mathrm{ml}^{-1}\right)$ stimulation (unpaired Student's $t$-test; ${ }^{*} P$ $<0.05,{ }^{* *} P<0.01,{ }^{* * *} P<0.001$; data represent mean \pm s.e.m (compared to unstimulated epithelium or stimulated co-culture) from 3-4 healthy donors, with 1 biological replicate per donor; $n=3-4$ ). (f) Sequential time-lapse microscopic views showing freshly isolated neutrophils being recruited to the surface of living (lung blood microvascular) endothelium, and rolling on the surface membrane, under physiological flow and shear stress $\left(1 \mathrm{dyne} \mathrm{cm}^{-2}\right)$ in the small airway chip when stimulated with poly I:C. Yellow arrowhead indicates a neutrophil that is captured from the flowing medium that then rolls over the endothelial surface in subsequent images, directly adjacent to an already bound neutrophil (neutrophils were live stained with CellTracker Red; arrow indicates direction of flow; times are indicated in seconds; bar, $20 \mu \mathrm{m}$; representative of 3 independent experiments performed on 3 different donors). (g) Fluorescence micrographs showing recruitment of Calcein AM-labeled circulating neutrophils (green) to the surface of the (lung blood microvascular) endothelium with or without poly I:C stimulation of the small airway chips for 6 hours (bar, $50 \mu \mathrm{m}$; representative of 3 independent experiments performed on 2 different donors).

\section{Figure 3. Modeling COPD exacerbations in the small airway-on-a-chip} Graphs showing effects on production of the cytokines, IL-8, M-CSF, RANTES, and IP10 by TLR stimulation with LPS $\left(10 \mu \mathrm{g} \mathrm{ml}^{-1}\right)$ or poly I:C $\left(10 \mu \mathrm{g} \mathrm{ml}^{-1}\right)$ in small airway chips lined by 3 to 5 weeks old hAECs obtained from healthy versus COPD patients (all the COPD patients had a history of smoking). Note that TLR activation by these simulants 
of bacterial and viral infection significantly induced IL-8 and M-CSF release only in COPD chips, whereas IP-10 and RANTES release increased in both healthy and COPD epithelia (unpaired Student's $t$-test; ${ }^{*} P<0.05,{ }^{* *} P<0.01$; data represent mean \pm s.e.m (compared to unstimulated) from 4 different healthy and 4 different COPD donors, with 1-3 biological replicates [chips] per donor; $n=5-10$; the variability between biological replicates of the same donor was minimal [s.e.m $<5 \%$ of mean]).

Figure 4. Pharmacological modulation of IL-13 induced asthmatic phenotype and COPD exacerbation-associated inflammation in the small airway

chip. (a) Immunofluorescence micrographic views of the differentiated epithelium cultured for 4 to 6 weeks at air liquid interface, stained for the goblet cell marker, MUC5AC (green) and DAPI (blue; bar, $50 \mu \mathrm{m}$; representative of 3 independent experiments performed from 3 donors) and (b) graphic depictions of total area covered by goblet cells, effects on production of the cytokines GM-CSF and G-CSF, and cilia beating frequency after exposure of the epithelium to IL-13 with or without Dexamethasone $(1 \mu \mathrm{M})$ or Tofacitinib $(1$ or $10 \mu \mathrm{M})$ for 8 days (unpaired Student's $t$-test; skewed cytokines data were log transformed before comparison; ${ }^{*} P<0.05,{ }^{* *} P<0.01$, ${ }^{* * *} P<0.001$; data represent mean \pm s.e.m (compared to IL-13-treated condition) from 3 healthy donors, with 1-2 biological replicates per donor; for goblet cell analysis 3-5 representative areas per condition were used for quantification; $n=3-9)$. (c) Fluorescence microscopic views showing recruitment of Hoechst-stained circulating neutrophils (green) to the surface of the pulmonary blood microvascular endothelium in the small airway-on-a-chip containing a differentiated COPD epithelium with an underlying endothelium pre-treated with $10 \mathrm{nM}$ Budesonide, $500 \mathrm{nM}$ BRD-4 inhibitor, or 
$0.1 \%$ DMSO diluent (untreated) for 24 hours prior to poly I:C $\left(10 \mu \mathrm{g} \mathrm{ml}^{-1}\right)$ stimulation (representative of 3 independent experiments using 3 different donors; bar, $50 \mu \mathrm{m}$ ). (d) A graphic depiction of the neutrophil adhesion results shown in c (black bars) compared with neutrophil recruitment data obtained when similar studies were carried out in static Transwell cultures (white bars) (unpaired Student's $t$-test; ${ }^{* * *} P<0.001$; data represent mean \pm s.e.m (chip against Transwell) from 2-4 different COPD donors, with 1-4 biological replicates per donor and 3-10 independent fields of view per chip or Transwell; $n=15-59)$. (e) Effects of (10 nM) Budesonide and (500 nM) BRD-4 inhibitor on expression of genes encoding the endothelial cell adhesion molecules, E-selectin, VCAM-1 and ICAM-1, compared to the untreated (0.1\% DMSO) group, as measured using real time PCR; human pulmonary blood microvascular cells were studied (unpaired Student's $t$-test; ${ }^{*} P<0.05,{ }^{*} P<0.01$, n.s. not significant; data represent mean \pm s.e.m from one of the donors studied in $\mathbf{d}, 3-4$ biological replicates per condition; $n=3-4)$.

Figure 5. Therapeutic modulation of inflammatory cytokine and chemokine production in the COPD small airway chip. Analysis of the effects of Budesonide and BRD-4 inhibitor on the secretion of IL-8, MCP-1, GRO $\alpha, \mathrm{IL}-6$, and GM-CSF into the microvascular channel of chips lined by COPD epithelium cultured at air liquid interface for 3 to 5 weeks when challenged with poly I:C (unpaired Student's $t$-test; ${ }^{*} P<0.05$, ${ }^{* *}$ $P<0.01,{ }^{* * *} P<0.001$; data represent mean \pm s.e.m (compared to untreated) from 3 different COPD epithelial and 2 different lung blood microvascular endothelial cell donors, with 1-4 biological replicates per donor; $n=6-8$ ). 


\section{Human airway Small airway-on-a-chip}

\section{Cell types}

$\begin{array}{llc}\text { Ciliated cells } & \sim 30 \% & \sim 20-30 \% \\ \text { Goblet cells } & \sim 10-15 \% & \sim 10-20 \% \\ \text { Club cells } & \sim 11-44 \% & \sim 25 \% \\ \text { Basal cells } & \sim 6-30 \% & \sim 20 \%\end{array}$

\section{Cilia}

\begin{tabular}{lcc} 
Structure & $9+2$ structure & $9+2$ structure \\
Length & $\sim 6 \mu \mathrm{m}$ & $\sim 6 \mu \mathrm{m}$ \\
Beating frequency & $9-20 \mathrm{~Hz}$ & $9-20 \mathrm{~Hz}$ \\
Transport velocity & $40-150 \mu \mathrm{m} / \mathrm{sec}$ & $40-100 \mu \mathrm{m} / \mathrm{sec}$ \\
\hline
\end{tabular}

Table 1. Comparison of the structure and function of the living human airway with the human small airway-on-a-chip. Human airway cell composition characteristics and properties of pulmonary cilia were obtained from prior publications ${ }^{21,}$ 23, 48, 49 . 


\section{ONLINE METHODS}

\section{Device fabrication}

The upper and lower layers of the microfluidic device (Figure 1A) were produced by casting polydimethylsiloxane (PDMS) pre-polymer on molds prepared using stereolithography (Fineline, USA). Curing (10:1 PDMS base to curing agent; w/w) was carried out overnight at $60^{\circ} \mathrm{C}$ to produce devices containing two adjacent parallel microchannels (top channel, 1,000 $\mu \mathrm{m}$ wide $\mathrm{Q} 1,000 \mu \mathrm{m}$ high; bottom channel, 1,000 $\mu \mathrm{m}$

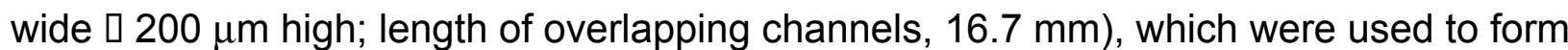
the airway lumen and microvascular channel, respectively. The channels were separated by a thin $(\sim 10 \mu \mathrm{m})$ semi-porous polyester membrane $(0.4 \mu \mathrm{m}$ pores $)$ that was purchased from Maine Manufacturing (USA). The membrane was laser cut, plasma-treated using Plasma Etcher PE-100 (Plasma Etch, USA) with one cycle of 50

Watts of oxygen gas ( 15 standard $\mathrm{cm}^{3} \mathrm{~min}^{-1}$ ) for $15 \mathrm{sec}$, sandwiched between the carefully aligned top and bottom channels (Fig. 1b), and treated with ultraviolet light in a UVO-Cleaner (Model 342; Jelight, USA) for 30 minutes before applying a collagen coating.

\section{Microfluidic cell culture}

Primary human airway epithelial cells (hAECs) obtained from commercial suppliers (Promocell, Germany; Lonza, USA, and Epithelix, Switzerland) were expanded in $75 \mathrm{~cm}^{2}$ tissue culture flasks using airway epithelial growth medium (BEBM; Catalog no. CC-3171) supplemented with growth factors/supplements (BulletKit Supplements; Lonza) until $70-80 \%$ confluent. Isolation of the lung epithelial cells, based 
on information provided by the vendors, had been carried out by dissecting airways from whole lungs or lung biopsies, subjecting them to enzymatic digestion and subsequently culturing them in a selective growth medium. Age and sex of healthy and COPD donors are described in Supplementary Table 2. All of the COPD patients, whose epithelial cell we studied in experiment in Fig. 3 had a history of smoking.

Prior to culturing the cells, the porous membrane of the device was coated on both sides with rat tail collagen type I (300 $\mathrm{g} \mathrm{m} \mathrm{m}^{-1}$; Corning, USA) at $37^{\circ} \mathrm{C}$ for 24 hours. The hAECs were then trypsinized and seeded onto the collagen-coated porous membrane in the upper channel of the device at a concentration of 2 to $5 \times 10^{6}$ cells $\mathrm{ml}^{-1}$ ( 2 to $5 \square 10^{5}$ cells $\mathrm{cm}^{-2}$, or 4 to $10 \square 10^{4}$ cells per chip) and allowed to attach under static conditions. Three hours later, the cell monolayer was washed with fresh medium and the cultures were maintained in submerged state until fully confluent (typically 4 to 5 days after seeding) under constant flow $\left(60 \mu \mathrm{L} \mathrm{h}{ }^{-1}\right)$ using an IPC-N series peristaltic pump (Ismatec, Switzerland). The chips were cultured in an incubator containing $5 \% \mathrm{CO}_{2}$ and $16-18 \% \mathrm{O}_{2}$ at $85-95 \%$ humidity.

When confluent, the apical medium was removed and an air-liquid interface (ALI) was generated to trigger differentiation. The hAECs were maintained at ALI for 3 to 5 weeks with constant flow of growth medium in the bottom channel, which was sufficient to support epithelial cell viability and function for weeks in culture. The apical surface of the epithelium was rinsed once weekly with growth medium to remove debris. To control for variability in our studies, we ensured that all cultures reached a similar level of morphological and functional differentiation before we carried out any experiments. In our initial characterization studies, we determined that the primary human epithelial cells 
formed a stable and terminally differentiated ciliated epithelium within 3 to 5 weeks after being placed under an air-liquid interface, as defined using non-invasive microscopic imaging to confirm formation of a confluent epithelial layer expressing actively beating surface cilia, and chips that failed to reach this standard by 5 weeks were discarded. We did not observe any notable difference between donors in our studies. Mucociliary transport was visualized using $1 \mu \mathrm{m}$ diameter fluorescent microbeads (Life Technologies, USA ) diluted in PBS, injected in the upper channel of a fully differentiated small airwayon-a-chip and imaged using a high speed Hamamastu ORCA-Flash 4.0 camera mounted on a Zeiss AxioObserver Z1 microscope. Permeability measurements were done by flowing Inulin (4 kDa)-FITC, Dextran (10 kDa)-Cascade Blue or Dextran (70 $\mathrm{kDa}$-Texas red diluted in growth medium $\left(100 \mu \mathrm{g} \mathrm{ml}^{-1}\right)$ at $60 \mu \mathrm{L} \mathrm{h}^{-1}$ in top channel for $24 \mathrm{~h}$ and measuring the fluorescence intensity of medium flowing out of the top and bottom channels in 3 individual small airway chips. The apparent permeability was calculated using the following formula:

$$
P_{a p p}=\frac{J}{A \cdot \Delta C}
$$

With $P_{a p p}=$ Apparent permeability, $J=$ Molecular flux $A=$ Total area of diffusion, and $\Delta C=$ Average gradient $(\sim 1$ because of the low flow rate $)$.

Once differentiation of the hAECs was accomplished, human lung microvascular endothelial cells (HMVECs; Lonza; purchased as 'lung blood microvascular' endothelial cells before they switched to the 'lymphatic + vascular' mix) were used for analysis of neutrophil adhesion and rolling, drug efficacy testing and experiments involving COPD chips. We performed flow cytometric analysis and confirmed that our endothelial cells were $>98 \%$ pure blood microvascular endothelial cells free of cells expressing 
lymphatic antigens (Lymphatic Vessel Endothelial Receptor 1 and Podoplanin). Human umbilical vein endothelial cells (HUVECs; Angio-proteomie; USA or Lonza) were used for synergistic cytokine secretion studies; both types of endothelial cells were seeded onto the lower side of the porous membrane through the bottom channel at a density of $2 \times 10^{7}$ cells ml ${ }^{-1}\left(2 \square 10^{5}\right.$ cells $\mathrm{cm}^{-2}$ or $4 \square 10^{4}$ cells per chip). Endothelial cells were seeded in Lonza EBM-2 endothelial cell basal growth medium (catalog no. CC-3156) supplemented with EGM-2MV SingleQuot Kit growth factors/supplements under static conditions with the chip oriented upside down to allow cell adhesion to undersurface of the porous membrane. Two hours later, flowing of growth medium $\left(60 \mu \mathrm{L} \mathrm{h}{ }^{-1}\right)$ was resumed until cells were fully confluent (usually 3 to 6 days after seeding). There was no significant difference in the cell densities in the epithelial or endothelial layers when we compared epithelium cultured alone or in the presence of endothelium.

\section{Immunofluorescence Microscopy}

To carry out immunofluorescence staining, cells were washed by flowing phosphate-buffered saline (PBS) through both the top and bottom channels, fixed with 4\% paraformaldehyde (Electron Microscopy Sciences, USA) for 15 minutes without flow, and washed gently by flowing additional PBS before being stored at $4^{\circ} \mathrm{C}$ until use. The cells cultured on-chip were washed again with PBS, permeabilized with $0.2 \%$ Triton X100 (Sigma) in PBS for 2 hours and exposed to blocking buffer composed of PBS containing 1\% BSA (Sigma) and 5\% fetal bovine serum (Life Technologies, USA)] for 30 minutes at room temperature. Cultures were stained for ciliated cells (anti- $\beta$-tubulin IV, 1:100, clone ONS.1A6; Genetex, Taiwan), goblet cells (anti-Mucin5AC, clone H-160, Santa Cruz Biotech., USA), basal cells (anti-Cytokeratin 5, Abcam, USA), tight junctions 
(anti-ZO1, clone 1A12; Life Technologies, USA), or PECAM-1 (clone WM-58;

eBioscience, USA). When cell adhesion molecules were stained on endothelial cells, the cells were not permeabilized to visualize surface antigens. In all studies, antibodies diluted in blocking buffer were introduced in the channels, incubated for 1 hour at room temperature or overnight at $4^{\circ} \mathrm{C}$ and cultures were washed with PBS three times each for 5 minutes at room temperature. Secondary antibodies (Life Technologies, USA) were incubated for 45 minutes at room temperature and washed three times with PBS. In some studies, TO-PRO-3 (Life Technologies, USA) was used to label nuclei following secondary antibody staining. Devices were then cut using a razor blade and membranes were delicately lifted from the PDMS and mounted on microscopy slides in mounting medium (Vectashield; Vector Laboratories, USA) with or without DAPI. Fluorescence imaging was carried out using confocal laser scanning microscopy (SP5 X MP DMI-6000, Germany). Image processing and 3-dimensional Z-stack reconstruction was done using Imaris (Bitplane, Switzerland) and ImageJ (downloaded from http://imagej.nih.gov/ii/) software. When using two primary antibodies of the same species, Zenon® Antibody Labeling Kit (Life Technologies; USA) was used to preconjugate the antibodies separately prior to addition to fixed cells. For IL-13 experiments, cultures were treated with IL-13 (Peprotech, USA), dexamethasone (Sigma-Aldrich, USA) or Tofacitinib, fixed after 8 days of treatment, and stained for MUC5AC and DAPI. Tofacitinib was purchased commercially (CP-690550, Selleckchem, USA) and not obtained from Pfizer, and the doses were optimized in-house.

Quantification of goblet cells hyperplasia was done by measuring the percent of the projected area covered by goblet cells in eight different fields for each condition. 


\section{Scanning and transmission electron microscopy}

For electron microscopic analysis, the porous membranes supporting the cell cultures were excised from the devices using a razor blade, and cells were fixed using $2.5 \%$ glutaraldehyde (Electron Microscopy Sciences, USA) in $1 \%$ sodium cacodylate (Sigma) for $1 \mathrm{~h}$ at room temperature. Fixed cells were rinsed with $1 \%$ sodium cacodylate and post-fixed with $1 \%$ osmium tetroxide (Electron Microscopy Sciences; USA) in $1 \%$ sodium cacodylate for 90 minutes in a fume hood. Cells were dehydrated sequentially in ethanol gradients, rinsed in hexamethyldisilazane, air dried overnight in a desiccator at room temperature and then mounted on a conductive carbon support, coated with gold and imaged with a VEGA III scanning electron microscope (Tescan, Czech Republic).

For transmission electron microscopy, fixed cells were and embedded in Taab 812 Resin (Marivac Ltd., Nova Scotia, Canada) and incubated at $60^{\circ} \mathrm{C}$ for one day. Samples were cut in $80 \mathrm{~nm}$ sections with Leica ultracut microtome, picked up on 300 mesh formvar-carbon coated Cu grids, stained with $0.2 \%$ Lead Citrate and viewed and imaged under the Philips Technai BioTwin Spirit Electron Microscope.

\section{Analysis of ciliated, goblet and basal cells, and mucociliary transport.}

Cilia beating frequency measurements were performed using a high speed Hamamatsu ORCA-Flash 4.0 camera mounted on a Zeiss AxioObserver Z1 microscope. The epithelium was washed with warm PBS before analysis to avoid mucus accumulation and slowing cilia beating. High-speed movies of beating cilia were recorded at approximately 200 frames per second and played at 30 frames per second for 1 second allowing manual counting. Four to five random areas of each chip were counted and cilia beating frequencies were averaged for each condition. For IL-13 
treatment experiments, cilia beating frequency was recorded and measured after 8 days of treatment ( $n=3-4$ chips). Epithelial cell types were quantified as previously described $^{1}$. Briefly, for ciliated cells and basal cells, fully differentiated epithelia from 3 small airway chips were trypsinized, centrifuged (1,000 $\mathrm{g}$ for $2 \mathrm{~min})$, air-dried for 1 hour at room temperature, fixed with $100 \%$ ice cold acetone, rinsed with PBS, stained for $\beta$-tubulin (ciliated cells marker) or Cytokeratin 5 (basal cells marker) and counterstained with DAPI. Ciliated cells and basal cells were counted in 6 fields per chip and percentages calculated. For goblet cells, fully differentiated epithelia from 4 chips were fixed with $4 \%$ paraformaldehyde and stained for MUC5AC (goblet cells marker). Quantification of goblet cells was done by measuring the percent projected area covered by MUC5AC staining in 5 different fields for each condition. Mucociliary transport was evaluated by measuring the displacement of $1 \mu \mathrm{m}$ polystyrene fluorescent beads diluted in PBS and introduced in the top channel of the small airway chip for 1 second as presented in Fig. 1k.

\section{Gene expression analysis}

Total RNA was extracted from differentiated epithelial or endothelial cells in situ on-chip using RNeasy Mini Kit (Qiagen, USA). The RNA was incubated with DNase I (Qiagen, USA) for 15 minutes at room temperature to remove residual contaminating genomic DNA, enzyme heat-inactivated at $65^{\circ} \mathrm{C}$ for 5 minutes, and then reverse transcribed into cDNA using SuperScript $₫$ Reverse Transcriptase III kit (Invitrogen, USA). cDNA synthesis was primed by mixing $1 \mu$ of oligodT $(50 \mu \mathrm{M})$ and $1 \mu \mathrm{l}$ of dNTP mix (10 mM) with up to $500 \mathrm{ng}$ total RNA. Real time PCR was carried out using a CFX96 real-time PCR system (Bio-Rad, USA). Reactions contained $2 \mu \mathrm{l}$ cDNA, $10 \mu \mathrm{l}$ 
2DUniversal SYBR® Green Supermix (Bio-Rad, USA) and $3 \mu \mathrm{l}$ of each forward and reverse primers (300 $\mathrm{nM}$ final concentration), and $2 \mu \mathrm{l}$ molecular biology-grade water, and results were quantified using $2^{-\Delta \Delta C t}$ method $^{2}$. The list of primers used is presented in Primers were either designed using online Primer3 application (http://frodo.wi.mit.edu/primer3/) or used from previous reports ${ }^{3}$ and sequences are depicted in the Supplementary Table 1.

\section{Analysis of chemokines and cytokines}

The effluent of flowing medium was analyzed for a panel of cytokines and chemokines (IL-8, IP-10, RANTES, IL-6, M-CSF, G-CSF, GM-CSF and GROa) using custom Milliplex assay kits (Millipore, USA). Analyte concentrations were determined according to the manufacturer's instructions, using a LuminexFlexMap 3D system coupled with a Luminex XPONENT software (Luminex, USA). For endothelium depletion experiments, basal secretions were collected for each condition and RANTES, IL-6 and IP-10 were measured at 24 hours after poly I:C (InvivoGen, USA) treatment. For TLR stimulation experiments, COPD and healthy epithelium were challenged with LPS $10 \mu \mathrm{g} \mathrm{ml}^{-1}$ or poly I:C $10 \mu \mathrm{g} \mathrm{ml}^{-1}$ for 1 hour and secreted IL-8, M-CSF, IP-10 and RANTES were measured 24 hours following treatment. For COPD drug studies, in chips containing co-cultures of differentiated COPD epithelium and microvascular pulmonary endothelium, the endothelial cells were treated with $10 \mathrm{nM}$ Budesonide (Sigma), $500 \mathrm{nM}$ of the BRD4 inhibitor (provided by Pfizer) or $0.1 \%$ DMSO diluent (Sigma) under flow (60 $\left.\mu \mathrm{L} \mathrm{h}^{-1}\right)$ through the vascular channel for 24 hours before poly I:C $\left(10 \mu \mathrm{g} \mathrm{ml}^{-1}\right)$ was delivered into the airway channel for 6 hours. The vascular effluents were then collected for cytokine/chemokine analysis. 


\section{Analysis of neutrophil adhesion}

Neutrophils were isolated from fresh human blood by two-step gradient sedimentation. The peripheral blood mononuclear cells (PBMCs) were removed from the polymorphonuclear (PMN) cell population using Ficoll (Stem Cell Technologies, Canada) density centrifugation and then the PMNs were isolated using a modified Percoll (Sigma) protocol. Flow cytometry analysis for CD15 and CD16 expression confirmed purity of neutrophil population over $93 \%$. Isolated neutrophils were then livestained for 30 minutes at $37^{\circ} \mathrm{C}$ using cell tracker red or Hoechst (Life Technologies, USA), re-suspended in RPMI containing 10\% FBS (v/v) (Life Technologies, USA) and used within $3 \mathrm{~h}$ for recruitment assays in chips. The chips were flipped upside down and

neutrophils $\left(1 \mathrm{~L} 10^{7}\right.$ cells $\left.\mathrm{ml}^{-1}\right)$ were flowed $\left(2.7 \mathrm{ml} \mathrm{h}^{-1} ; 1\right.$ dyne $\left.\mathrm{cm}^{-2}\right)$ through the microvascular channel of the device to mimic the physiological hemodynamic conditions that exist in human post-capillary venules ${ }^{31}$. After 10 minutes, unbound neutrophils were washed away by flowing cell-free RPMI 10\% FBS medium for 5 minutes and micrographs of 4-5 random areas were taken for subsequent counting; quantification was done by counting attached neutrophils using ImageJ (http://imagej.nih.gov/ij/) and CellProfiler (http://www.cellprofiler.org) software. For experiments using Transwells, the

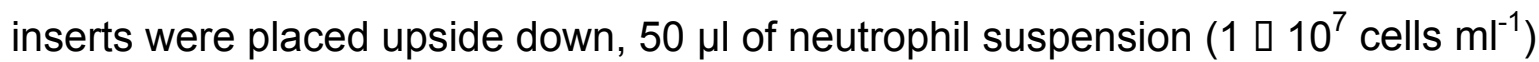
added gently to endothelium on the undersurface of each insert and incubated for 10 $\min$ at $37^{\circ} \mathrm{C}$. The inserts were then reverted to upright position in cell culture medium for 5 min to remove unbound neutrophils and were subsequently imaged and analyzed as chips.

\section{Statistical analysis}


All results and error bars are presented as mean \pm standard error of the mean (s.e.m). Data were analyzed with an unpaired Student's $t$-test using Graphpad Prism (GraphPad Software Inc., San Diego, CA, USA) or Excel software (Microsoft, USA). Differences between groups were considered statistically significant when $P<0.05$ ( $P$ $\left.<0.05,{ }^{* *} P<0.01,{ }^{* * *} P<0.001\right)$. Variability between biological replicates, for example in Fig. 3, of the same donor was minimal (s.e.m $<5 \%$ of mean). 


\section{METHODS REFERNCES}

1. Villenave, R. et al. In vitro modeling of respiratory syncytial virus infection of pediatric bronchial epithelium, the primary target of infection in vivo. Proceedings of the National Academy of Sciences of the United States of America 109, 50405045 (2012).

2. Livak, K.J. \& Schmittgen, T.D. Analysis of relative gene expression data using real-time quantitative PCR and the 2(-Delta Delta C(T)) Method. Methods 25, 402-408 (2001).

3. Benam, K.H., Kok, W.L., McMichael, A.J. \& Ho, L.P. Alternative spliced CD1d transcripts in human bronchial epithelial cells. PloS one 6, e22726 (2011). 


\section{a}

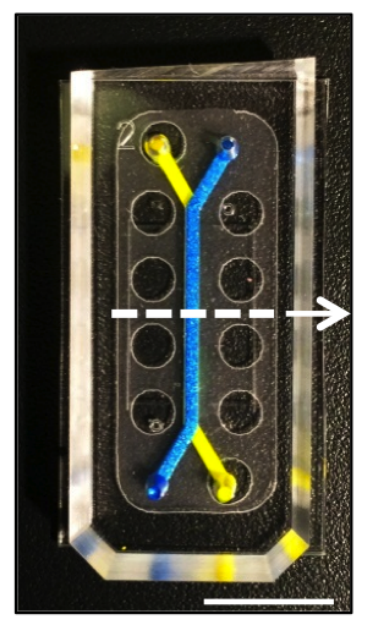

b

d

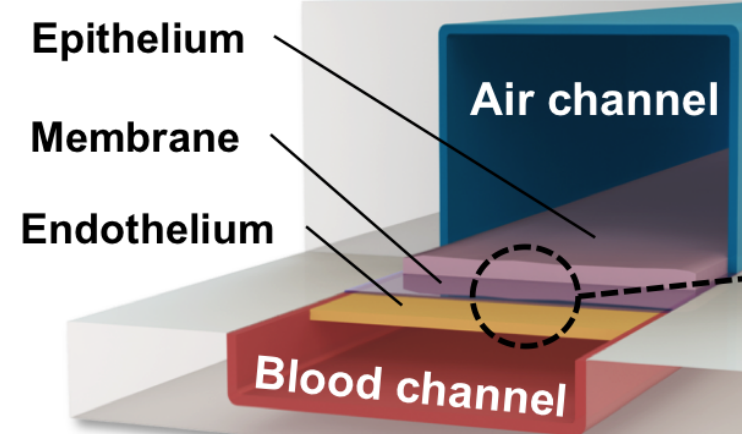

C
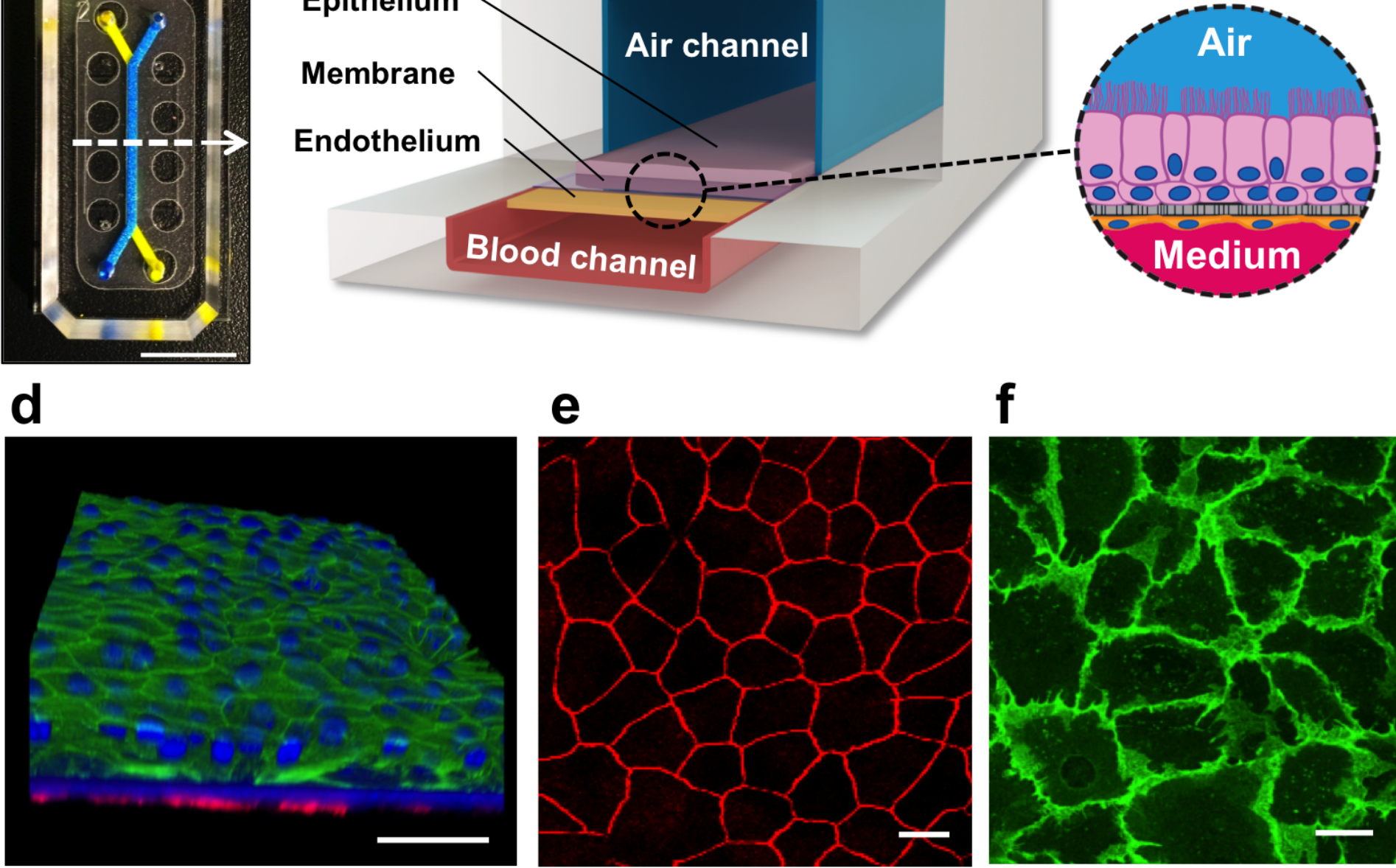

f

\section{g}

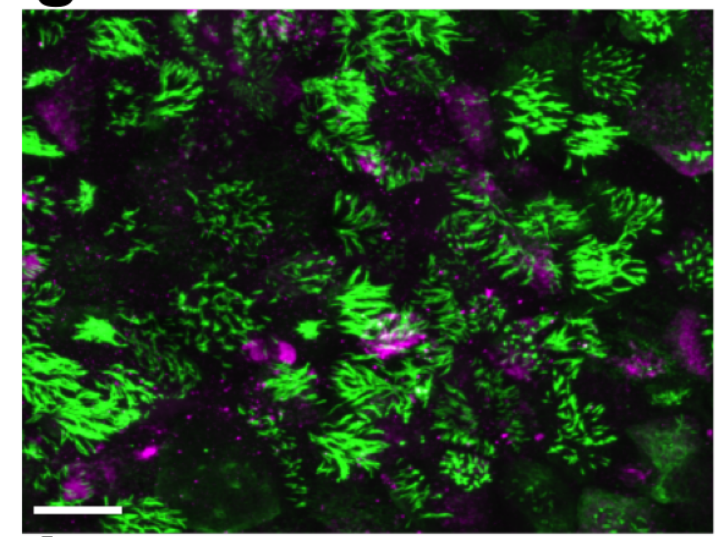

j
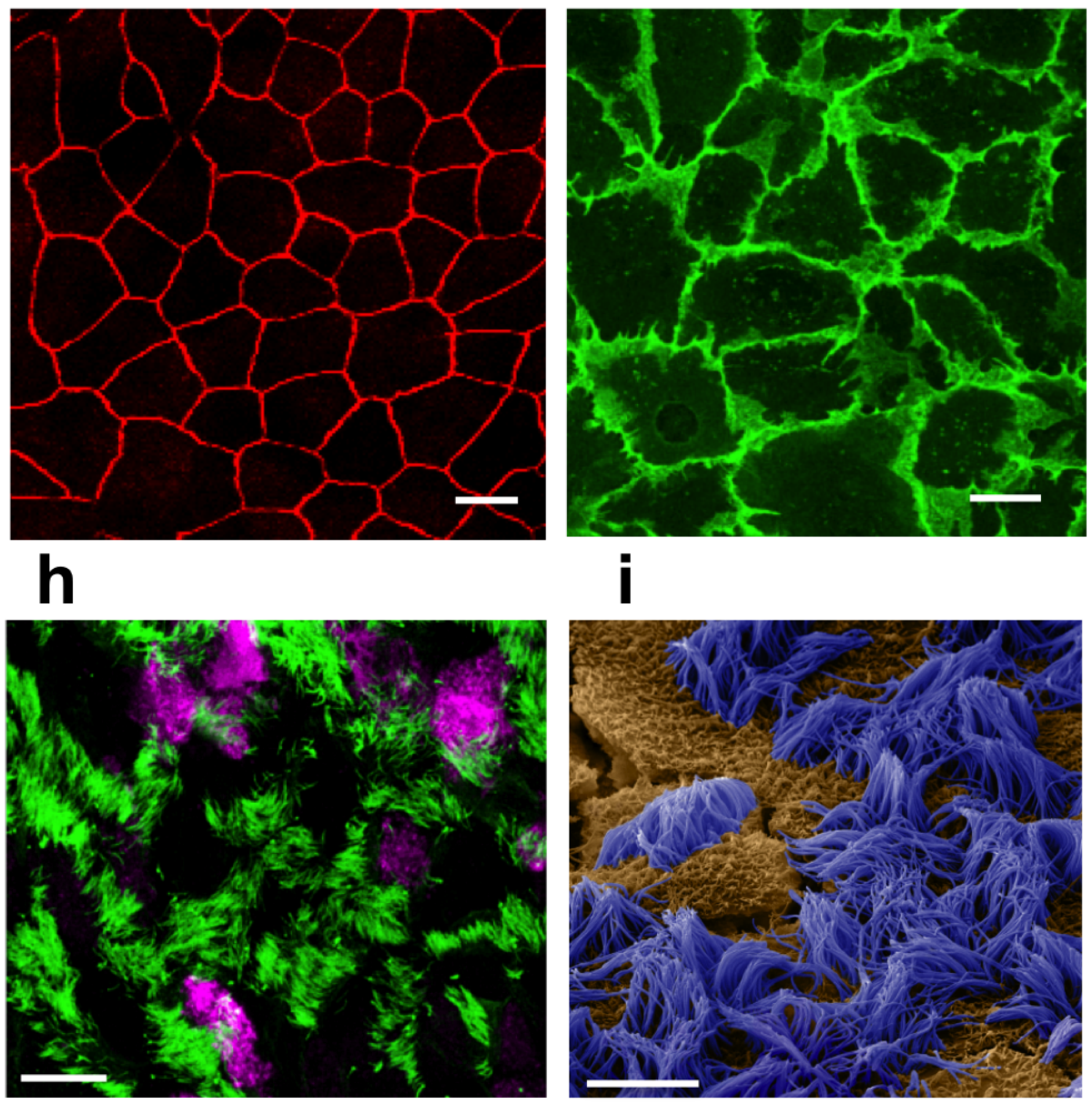

i
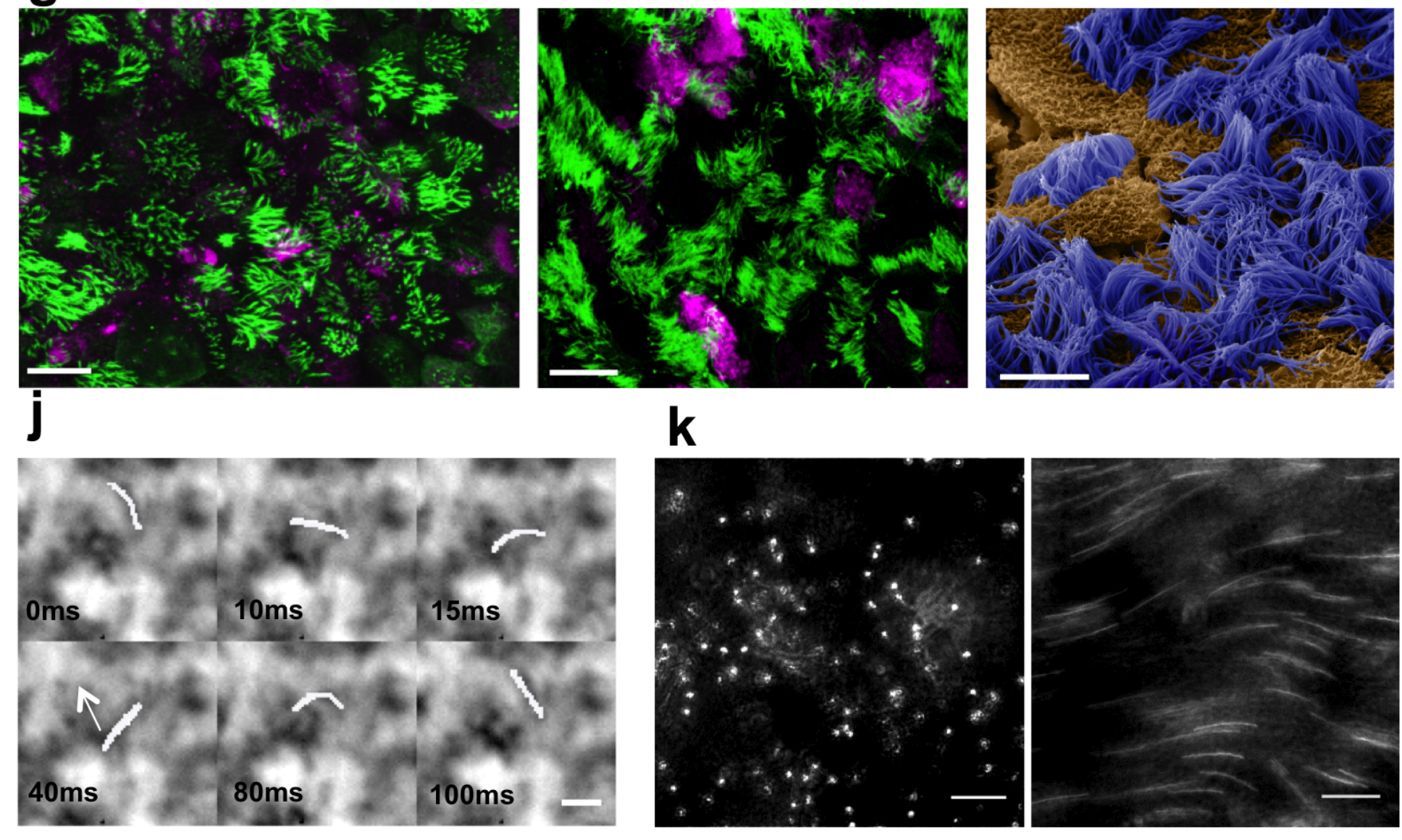

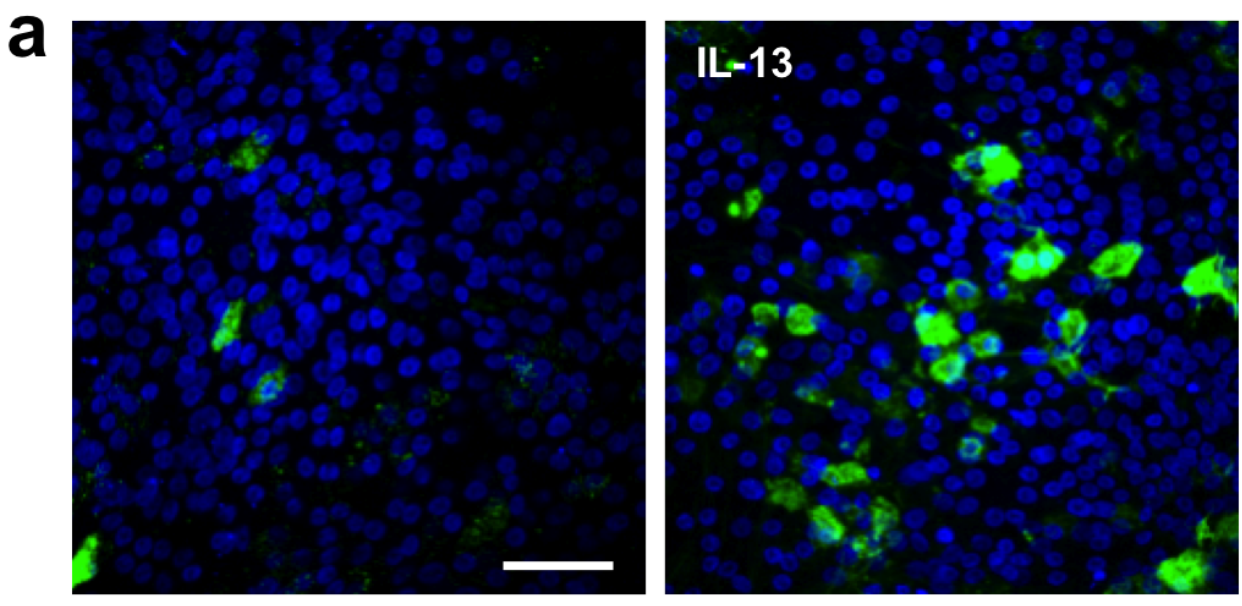

b

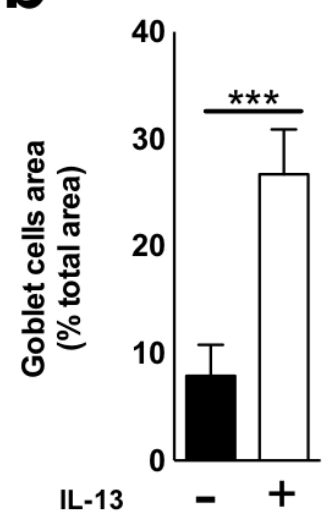

f
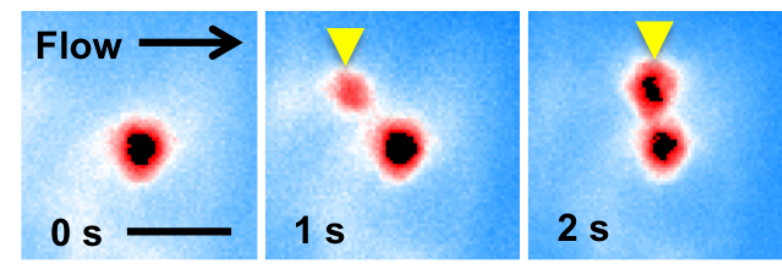
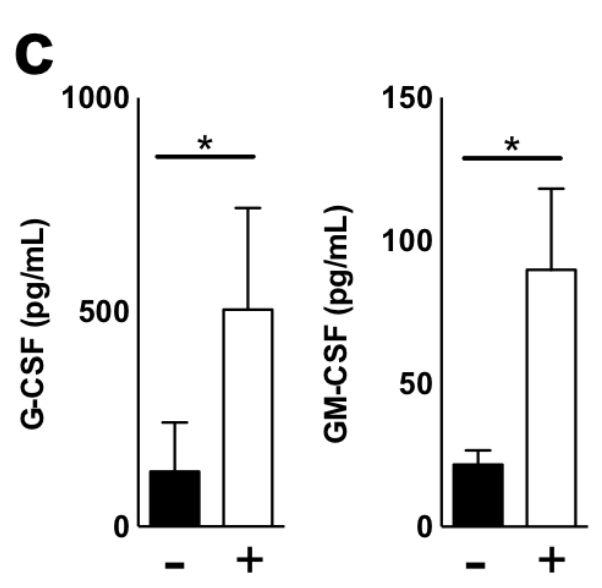

d

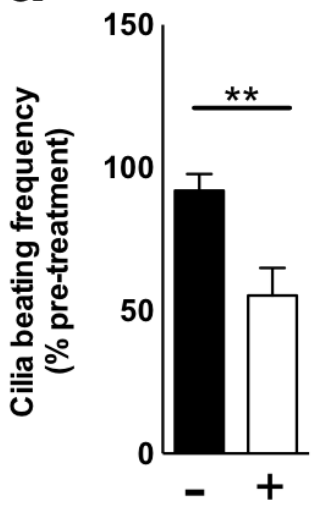

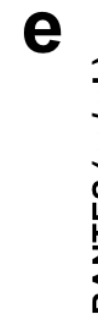
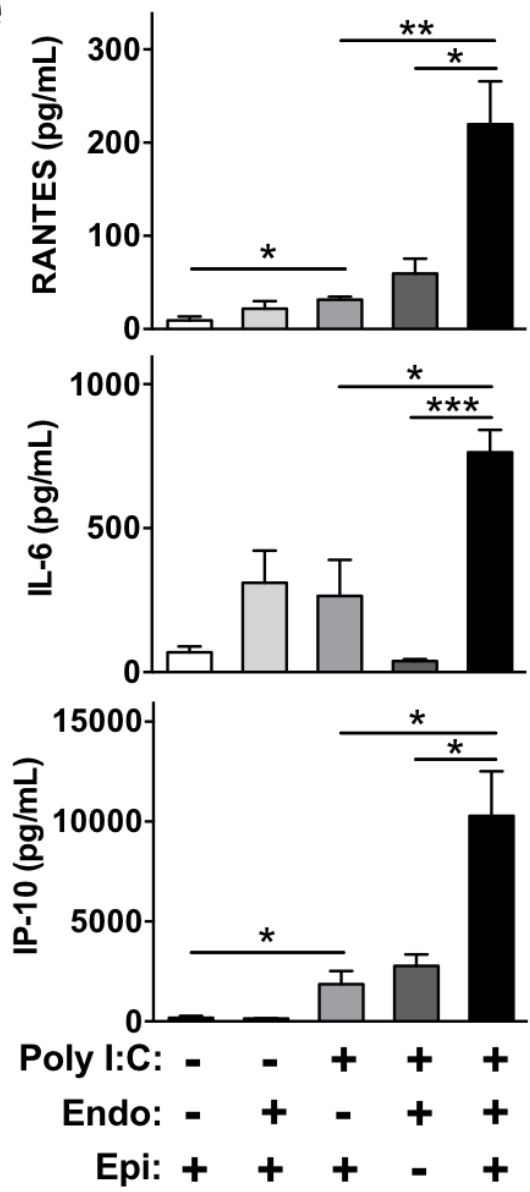

g
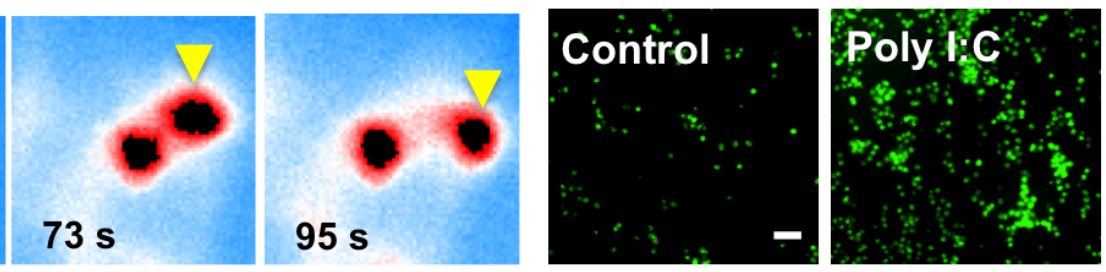

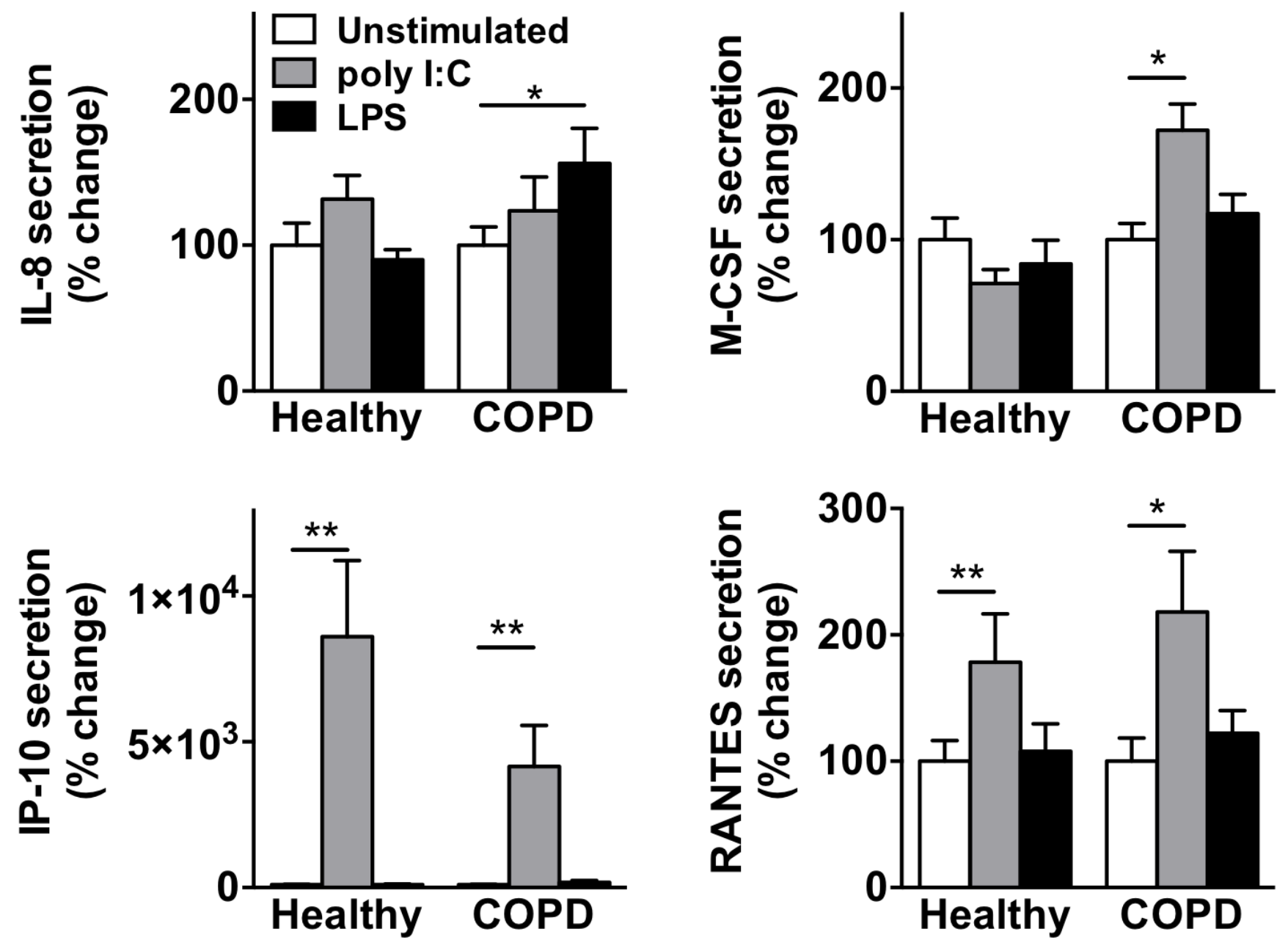


\section{a}
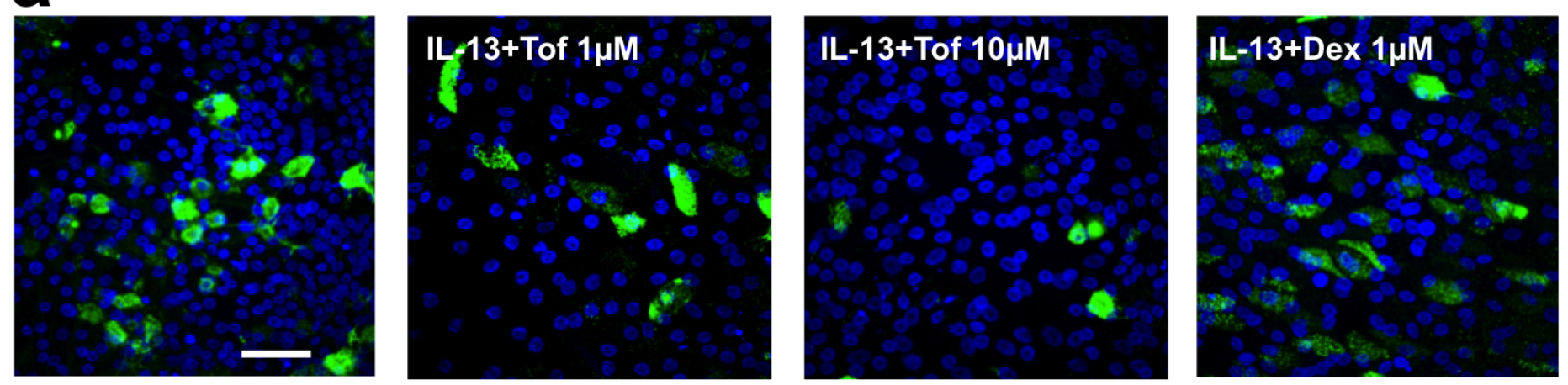

b

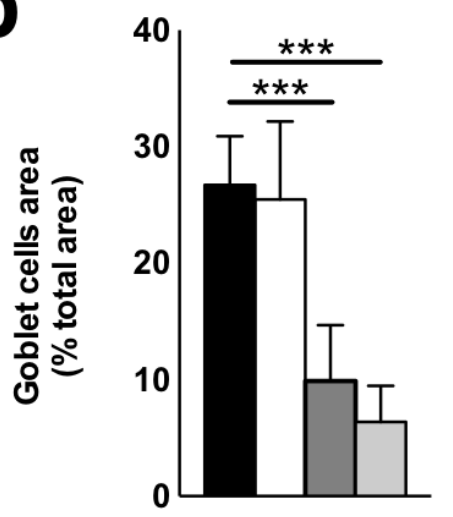

$$
\operatorname{Dex}_{(1 \mathrm{MM}):} \quad \mathbf{+}+-
$$

Tofacitinib $_{(1 \mu \mathrm{M}):}:-\boldsymbol{+}-$

Tofacitinib $_{(10 \mu \mathrm{M})}:=-\quad+$

C

Untreated

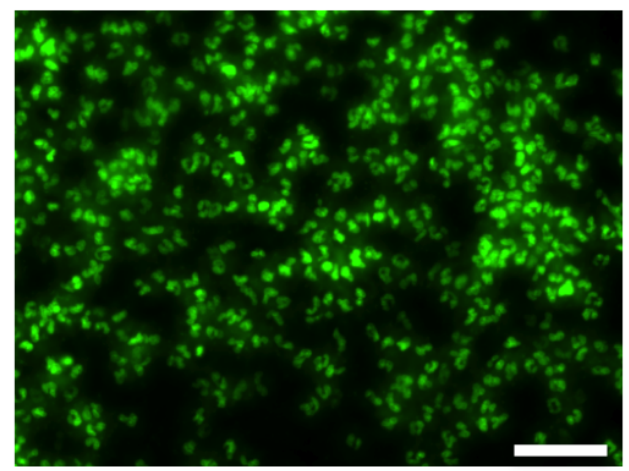

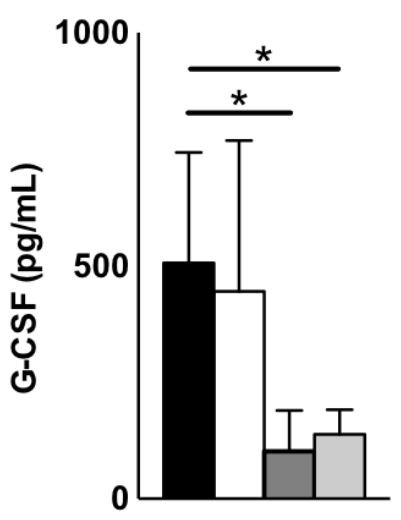

- + -

$-+$

- - +

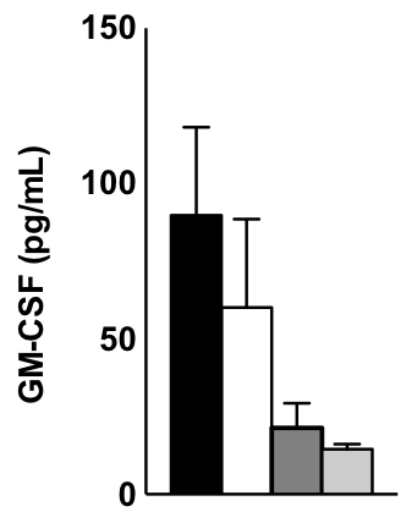

$-+--$

$-+-$

- - +

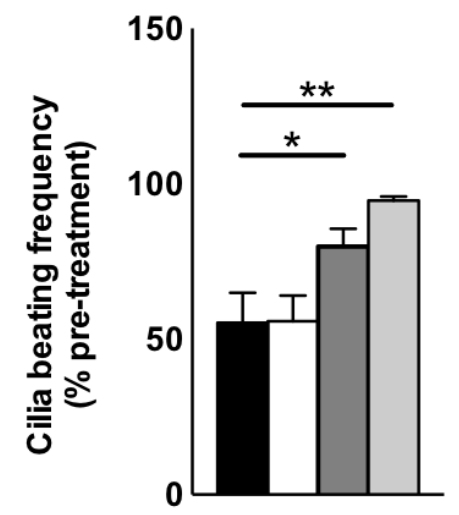

- + -

$-+$

- - +
Budesonide

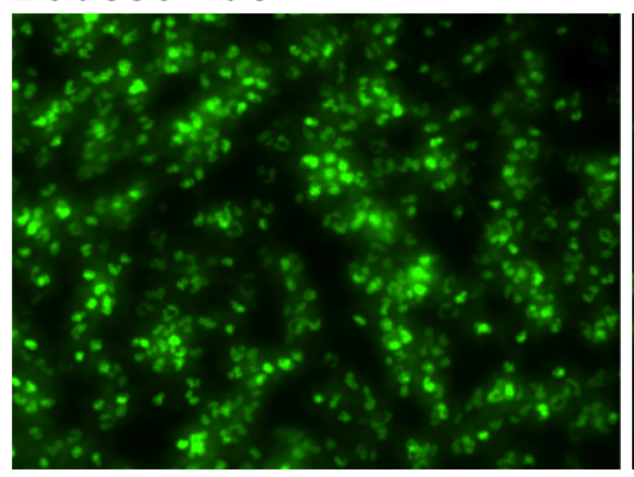

\section{BRD-4 Inhibitor}

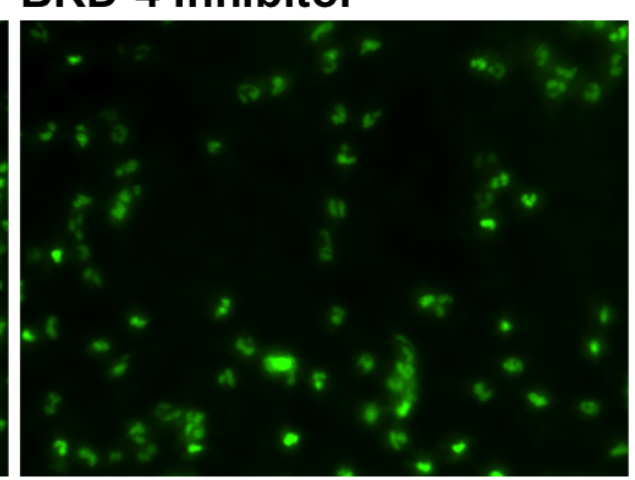

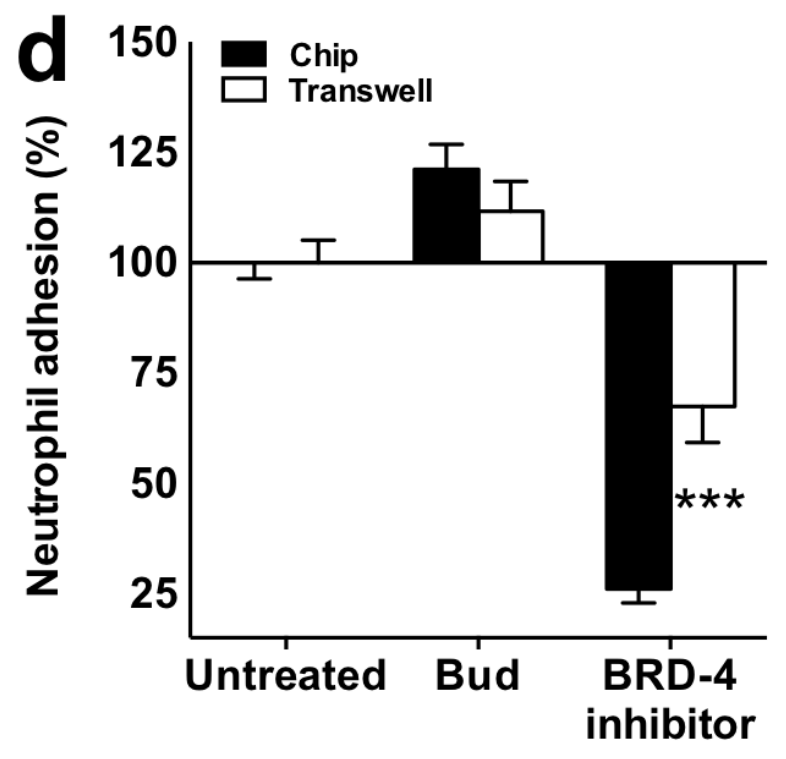

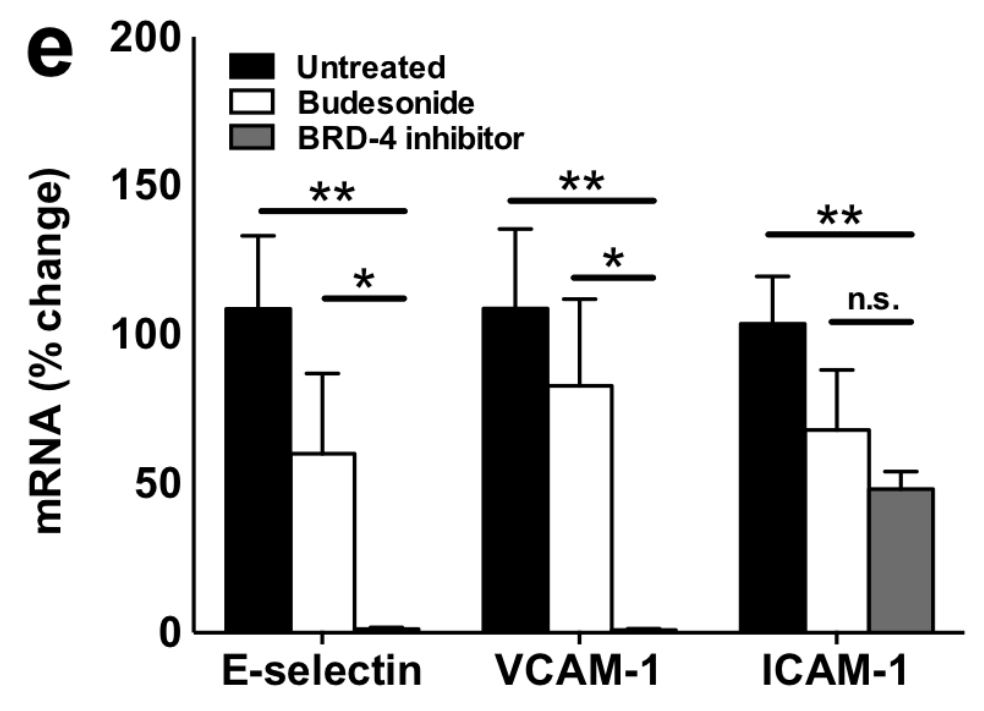



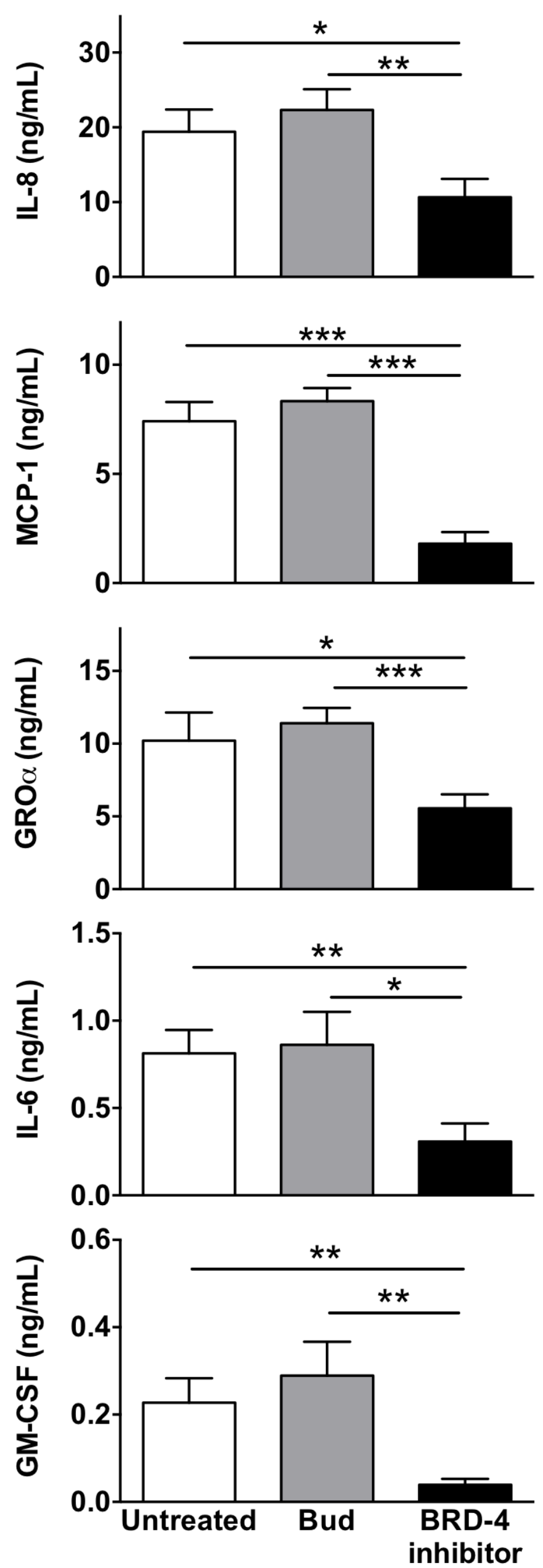


\section{SUPPLEMENTARY INFORMATION}

\section{Small airway-on-a-chip enables analysis of human lung inflammation and therapeutic responses in vitro}

Kambez H. Benam ${ }^{1,8}$, Remi Villenave ${ }^{1,8}$, Carolina Lucchesi ${ }^{1,7}$, Antonio Varone ${ }^{1,7}$, Cedric Hubeau $^{2}$, Hyun-Hee Lee ${ }^{3}$, Stephen E. Alves ${ }^{3}$, Michael Salmon ${ }^{3}$, Thomas C. Ferrante ${ }^{1}$, James C. Weaver ${ }^{1,4}$, Geraldine A. Hamilton ${ }^{1,7}$, Anthony Bahinski ${ }^{1}$, and Donald E. Ingber ${ }^{1,4-61}$

${ }^{1}$ Wyss Institute for Biologically Inspired Engineering, Harvard University, Boston, Massachusetts, USA; ${ }^{2}$ Pfizer, Cambridge, Massachusetts, USA; ${ }^{3}$ Merck Research Laboratories, Boston, Massachusetts, USA; ${ }^{4}$ Harvard School of Engineering and Applied Sciences, Cambridge, Massachusetts, USA; ${ }^{5}$ Vascular Biology Program, Boston Children's Hospital, Boston, Massachusetts, USA; ${ }^{6}$ Harvard Medical School, Boston, Massachusetts, USA; ${ }^{7}$ Current address: Emulate Inc., Cambridge, Massachusetts, USA; ${ }^{8}$ These authors contributed equally to this work.

\section{SUPPLEMENTARY DISCUSSION}

While other lung airway microfluidic models have been reported ${ }^{24,1}$ they were not able to produce a fully differentiated, pseudostratified, mucociliary epithelium, nor were they used to model complex inflammatory diseases of the lung, test therapeutics or analyze recruitment of circulating immune cells in vitro. In contrast, we were able to reconstitute the structure and function of a fully differentiated airway epithelium with an underlying microvascular endothelium, and by leveraging the ability of the microfluidic small airway-on-a-chip to study recruitment of circulating immune cells, to image 
endothelium-leukocyte interactions in real-time and quantitatively analyze neutrophil attachment under physiologically relevant flow conditions in vitro. While cyclic mechanical stretching is critical to recapitulate alveolar functions on-chip ${ }^{19,20}$, we found that providing a $1 \mathrm{~mm}$ tall channel to permit formation of a thick mucociliary epithelium and maintaining an air-liquid interface for at least three weeks in culture was sufficient to support differentiation of the human primary airway epithelial cells. We did not include pulmonary fibroblasts and smooth muscle cells that are known to contribute to defective alveolar regeneration and repair through deregulated ECM remodeling in $\mathrm{COPD}^{2}$, and to bronchospasm through hyper-contractile airway responses in asthma ${ }^{3}$; however, these connective tissue cells play a less pronounced role in driving airway inflammatory responses that were the focus of this study, and that are the major target for development of new anti-inflammatory therapeutics. Nevertheless, these other cell types could easily be integrated into the system in the future. In addition, the airway lumen channel of the chip could enable testing of new aerosolized therapeutics where local delivery to epithelium is desired, and permit analysis of other known triggers of clinical exacerbations of these diseases, including airborne particulates.

Our studies analyzing neutrophil rolling adhesion and capture, which are the most critical for assessing endothelial inflammatory function, utilized human pulmonary microvascular endothelium, while we used human umbilical vein endothelial cells in some of the other studies. Notably, both types of endothelium exhibited comparable cytokine secretion profiles to TLR challenge as evident by similar IL-6 release (Figs. $2 \mathbf{e}$ and Fig. 5). The contribution of COPD-derived endothelium to inflammatory responses also could be studied using this new method in the future. 
One example of the power of this method was the discovery that M-CSF may represent a new biomarker for COPD exacerbations induced by respiratory viruses. Identification of other biomarkers of clinical exacerbations in COPD patients, such as tumor necrosis factor alpha, C-reactive protein, or neutrophil-associated inflammatory molecules (e.g. IL-8, myeloperoxidase, elastase) ${ }^{4}$ were based on analysis of sputum or serum. Thus, our results represent the first biomarker that has been identified in vitro and, to our knowledge, M-CSF is the first to offer the possibility of discriminating between viral and bacterial causes of COPD exacerbations. This is important because current treatment for acute COPD exacerbations involves administration of systemic corticosteroids and antibiotics; hence, the availability of highly specific biomarkers could potentially stratify patients and help guide therapy by preventing unnecessary antibiotic use and subsequent development of bacterial resistance ${ }^{5}$.

Interestingly, the finding that epithelial production of M-CSF and IL-8 was increased in COPD chips compared to chips lined by normal epithelium when challenged with the same dose, time of exposure and route of administration of LPS and poly I:C indicates that the COPD cells exhibit a higher sensitivity (lower threshold for immune activation) compared with healthy normal cells. In other words, the healthy epithelium is indeed more tolerant to these pathogenic stimuli, which is consistent with the emerging concept in immunology of tolerance or resilience ${ }^{6}$. It is also in line with previous observation that COPD patients exhibit exaggerated inflammation and immune responses ${ }^{7}$. Thus, it is likely that a higher dose of LPS or poly I:C is required to elicit similar pro-inflammatory profile in healthy cells. 
The epithelium in our COPD chips exhibited a differentiated phenotype similar to that seen in chips created with healthy epithelium (Fig. 19, h), which is expected because both cell types were cultured in the same medium supplemented with retinoic acid that has been shown to suppress squamous metaplasia in culture ${ }^{11}$. Also, our finding that COPD cells recapitulate the inflammatory hyper-reactivity of airway epithelial cells observed in human COPD patients even though they do not exhibit squamous hyperplasia provides another great example of how use of a synthetic in vitro model such as this small airway-on-a-chip can permit one to dissect independent contributors to complex pathophysiological processes.

We also demonstrated that this new method can be used to analyze responses to therapy in vitro. Treatment of the small airway-on-a-chip with Tofacitinib, a pan-JAK inhibitor used for treatment of rheumatoid arthritis, reversed IL-13-induced phenotype to healthy levels, and this too is consistent with multiple studies that have shown that Tofacitinib or other JAK inhibitors (e.g., encapsulated pyridone 6, R256) suppress inflammation in animal models of allergy or asthma ${ }^{41}$. But because of the simplicity of our in vitro airway disease model, we gained new insight into the mechanism of action of this drug. For example, we discovered for the first time that Tofacitinib has a direct effect on the human airway epithelium, as evidenced by inhibition of IL-13-induced goblet cell hyperplasia, cytokine (G-CSF and GM-CSF) secretion, and reduction of cilia beating frequency. In addition, we could recapitulate the hyper-responsiveness of the epithelium in terms of TLR-induced chemokine release in the on-chip COPD model as well as mimic clinically relevant disease exacerbations. All of these mechanisms could be studied in greater detail in vitro using this approach in the future. 
Finally, using the human airway on a chip, we were able to identify a novel therapeutic anti-inflammatory agent in the form of a BRD4 inhibitor that reduces neutrophil capture by inhibiting NFKB transcriptional activity, which could potentially be useful for treatment of COPD exacerbations. In our study, this BRD4 inhibitor proved superior to a corticosteroid drug currently prescribed in clinic (budesonide) in terms of suppressing inflammatory cytokine secretion, reducing endothelial activation and expression of Eselectin, and thereby inhibiting associated neutrophil adhesion and rolling, which only can be studied under flow in vitro using this new method. The physiological relevance of these results is also supported by the finding that another small-molecule BRD4 inhibitor (JQ1) similarly exhibits greater anti-inflammatory effects than the glucocorticoid dexamethasone in vivo ${ }^{44,47}$.

There is also an unprecedented need to discover new effective anti-inflammatory therapeutics for chronic respiratory inflammatory disorders, such as COPD and asthma. Thus, given the inability of current in vitro models to co-culture human airway endothelium with microvascular endothelium and circulating leukocytes under physiological flow, the human small airway-on-a-chip represents a valuable new tool to facilitate in vitro analysis of human pulmonary disease mechanisms and advance preclinical drug discovery, as well as to study normal airway physiology. 


\section{SUPPLEMNTARY TABLES}

\begin{tabular}{|c|c|c|}
\hline Gene & Primer & Sequence ( 5 è $\left.3^{\prime}\right)$ \\
\hline E-selectin & $\begin{array}{l}\text { Forward } \\
\text { Reverse }\end{array}$ & $\begin{array}{l}\text { TGAAGCTCCCACTGAGTCCA } \\
\text { AGCTGCTGGCAGGAACAAAT }\end{array}$ \\
\hline VCAM-1 & $\begin{array}{l}\text { Forward } \\
\text { Reverse }\end{array}$ & $\begin{array}{l}\text { CAGTTGAAGGATGCGGGAGT } \\
\text { GCACGAGAAGCTCAGGAGAAA }\end{array}$ \\
\hline ICAM-1 & $\begin{array}{l}\text { Forward } \\
\text { Reverse }\end{array}$ & $\begin{array}{l}\text { GAGGGCACCTACСTCTGTCG } \\
\text { CСTGCAGTGCCСАTTATGAC }\end{array}$ \\
\hline MCP-1 & $\begin{array}{l}\text { Forward } \\
\text { Reverse }\end{array}$ & $\begin{array}{l}\text { GATCTCAGTGCAGAGGCTCG } \\
\text { TGCTTGTCCAGGTGGTCCAT }\end{array}$ \\
\hline IL-8 & $\begin{array}{l}\text { Forward } \\
\text { Reverse }\end{array}$ & $\begin{array}{l}\text { CCACCCСАAАTTTATCAAAGAA } \\
\text { CAGACAGAGCTCTCTTCCATCA }\end{array}$ \\
\hline IL-6 & $\begin{array}{l}\text { Forward } \\
\text { Reverse }\end{array}$ & $\begin{array}{l}\text { AGGAGACTTGCCTGGTGAAA } \\
\text { GTCAGGGGTGGTTATTGCAT }\end{array}$ \\
\hline GROa & $\begin{array}{l}\text { Forward } \\
\text { Reverse }\end{array}$ & $\begin{array}{l}\text { TCACCCCAAGAACATCCAAA } \\
\text { CTATGGGGGATGCAGGATTG }\end{array}$ \\
\hline HPRT & $\begin{array}{l}\text { Forward } \\
\text { Reverse }\end{array}$ & $\begin{array}{l}\text { GACTTTGCTTTCCTTGGTCAGG } \\
\text { AGTCTGGCTTATATCCAACACTTCG }\end{array}$ \\
\hline
\end{tabular}

Supplementary Table 1. Primer sequences used for real time quantitative PCR analysis. 


\begin{tabular}{|ccc|}
\hline & Age of donor (y) & Sex \\
\hline & 68 & Male \\
73 & Male \\
& 79 & Male \\
& 47 & Male \\
& 71 & Male \\
& 32 & Female \\
& 50 & Male \\
Healthy & 64 & Male \\
& 81 & Male \\
& 63 & Male \\
& 63 & Male \\
& 67 & Female \\
& 78 & Male \\
& 70 & Female \\
& 54 & Female \\
& 55 & Female \\
\hline COPD & 68 & Male \\
& 52 & Male \\
& 52 & Male \\
& 72 & Male \\
& 57 & Female \\
\hline Mean age \pm SD & $62 \pm 12$ & \\
\hline
\end{tabular}

Supplementary Table 2. Gender and age distribution of human lung epithelial cell donors. 


\section{SUPPLEMENT-ONLY REFERENCES}

1. Stucki, A.O. et al. A lung-on-a-chip array with an integrated bio-inspired respiration mechanism. Lab on a chip 15, 1302-1310 (2015).

2. Plantier, L., Boczkowski, J. \& Crestani, B. Defect of alveolar regeneration in pulmonary emphysema: role of lung fibroblasts. International journal of chronic obstructive pulmonary disease 2, 463-469 (2007).

3. Hirst, S.J. Airway smooth muscle as a target in asthma. Clinical and experimental allergy : journal of the British Society for Allergy and Clinical Immunology 30 Suppl 1, 54-59 (2000).

4. Koutsokera, A., Kostikas, K., Nicod, L.P. \& Fitting, J.W. Pulmonary biomarkers in COPD exacerbations: a systematic review. Respiratory research 14, 111 (2013).

5. Laue, J., Reierth, E. \& Melbye, H. When should acute exacerbations of COPD be treated with systemic corticosteroids and antibiotics in primary care: a systematic review of current COPD guidelines. NPJ primary care respiratory medicine 25 , 15002 (2015).

6. Ayres, J.S. \& Schneider, D.S. Tolerance of infections. Annual review of immunology 30, 271-294 (2012).

7. Hill, A.T., Campbell, E.J., Bayley, D.L., Hill, S.L. \& Stockley, R.A. Evidence for excessive bronchial inflammation during an acute exacerbation of chronic obstructive pulmonary disease in patients with alpha(1)-antitrypsin deficiency (PiZ). American journal of respiratory and critical care medicine 160, 1968-1975 (1999). 
a
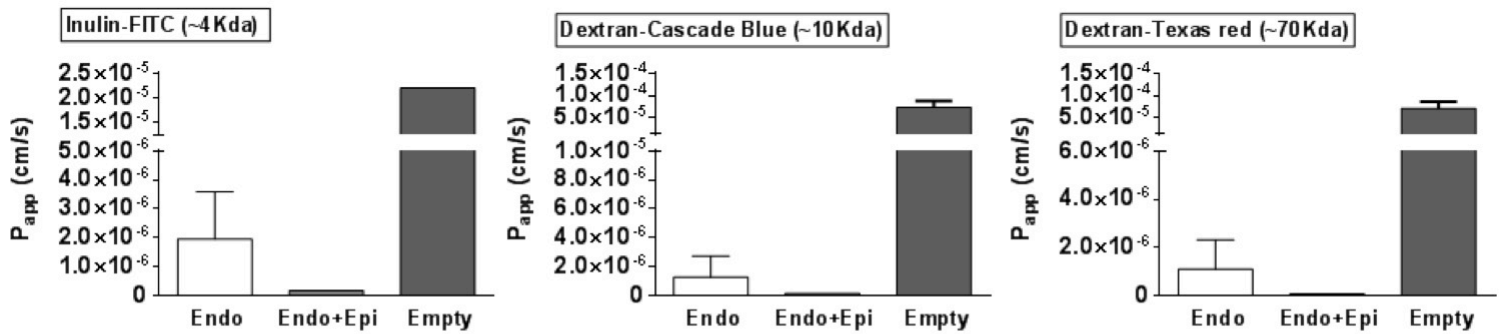

b

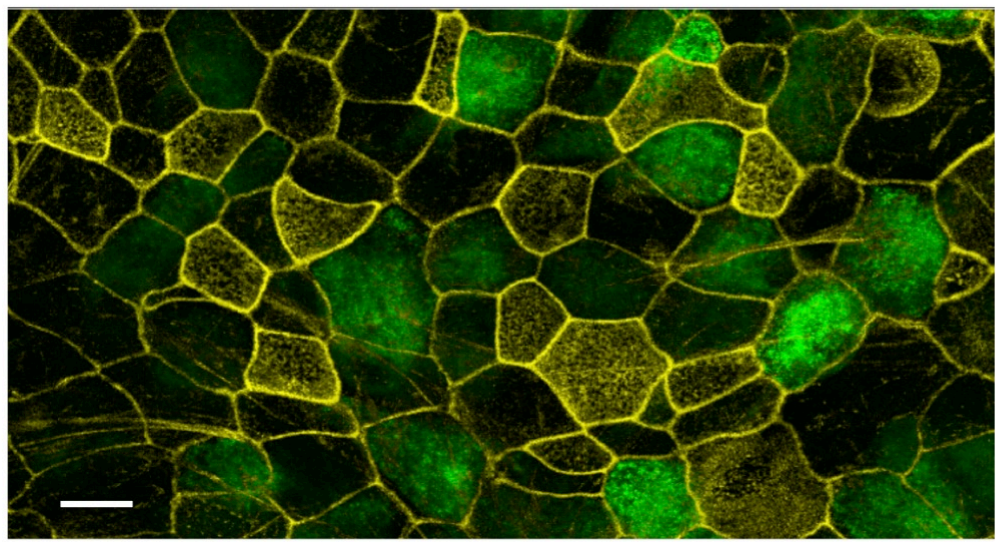

C

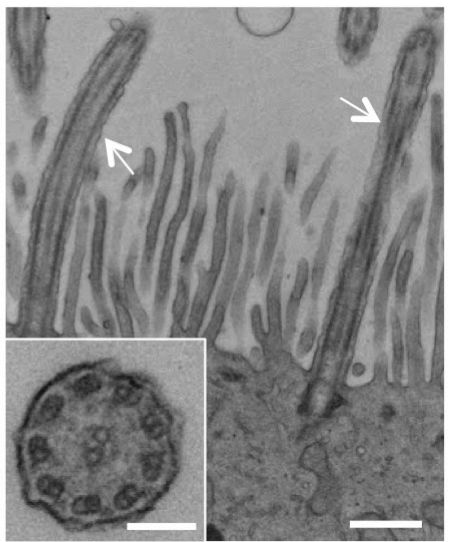

\section{Supplementary Figure 1}

Reconstitution of a differentiated human bronchiolar epithelium and pulmonary barrier on-chip

a) Epithelial barrier function was assessed by flowing Inulin-FITC ( 4 kDa), Dextran-Cascade Blue (10 kDa) or Dextran-Texas red (70 kDa) $\left(100 \mu \mathrm{m} \mathrm{ml}^{-1}-60 \mu \mathrm{L} \mathrm{h}{ }^{-1}\right)$ for $24 \mathrm{~h}$ through the epithelial side of the small airway-on-a-chip containing endothelial cells alone or cocultured with well-differentiated hAECs or no cells, and measuring fluorescence in the effluent from the top and bottom channels. Barrier permeability is presented as apparent permeability $\left(P_{a p p}\right.$; data from 1-2 independent biological replicates from 2 different donors are presented). b) A confocal immunofluorescence image of Club cells in bronchiolar epithelial cells differentiated on-chip (green, Club cell secretory protein 10; yellow, F-actin; bar, $20 \mu \mathrm{m}$; representative image from two independent stainings). c) Transmission electron micrographic views of cilia formed on the apical surface of human airway epithelial cells grown in the small airway-on-a-chip; white arrows indicate two cilia (bar, $200 \mathrm{~nm}$ ); inset shows a cross section of an axoneme at higher magnification, highlighting the typical 9+2 structure (bar, $100 \mathrm{~nm}$; representative image of 4 independent experiments performed in 4 different donors). 
a
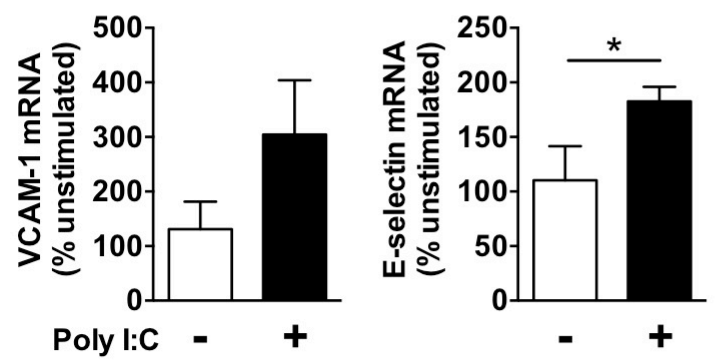

b

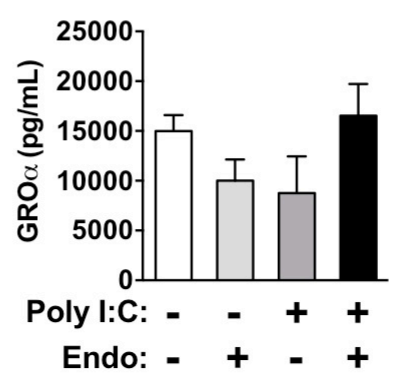

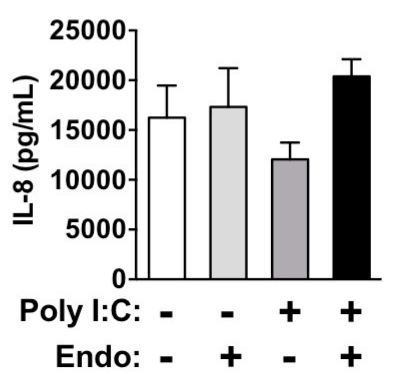

C
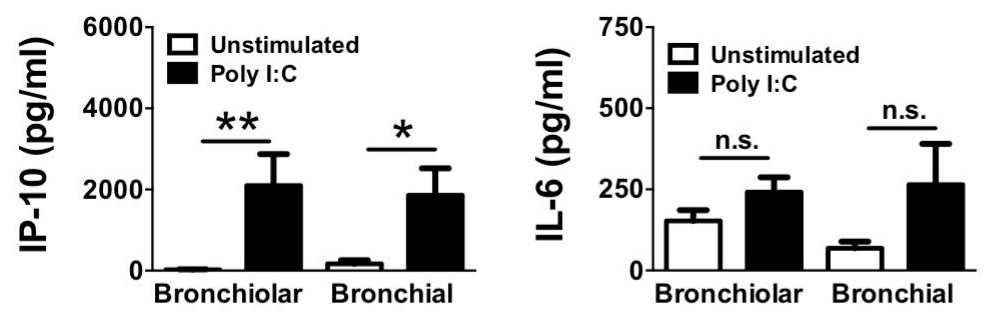

\section{Supplementary Figure 2}

Analysis of effects of the viral mimic poly I:C on interactions between epithelium and endothelium in the human small airway chip

a) Quantitative RT-PCR analysis of the effect of poly I:C $\left(10 \mu \mathrm{g} \mathrm{ml}{ }^{-1}\right)$ stimulation of the small airway chip for 6 hours on expression of endothelial genes encoding the cell adhesion molecules VCAM-1 and E-Selectin (unpaired Student's $t$-test; data represent mean \pm s.e.m (compared to unstimulated) from one donor, with 3 biological replicates per condition; $n=3$ ). b) GROa and IL-8 levels measured in basal secretions collected within the vascular effluent $24 \mathrm{~h}$ after fully differentiated hAECs cultured with (+) or without $(-)$ endothelial cells in the presence $(+)$ or absence $(-)$ of $10 \mu \mathrm{g} \mathrm{ml}^{-1}$ poly I:C (unpaired Student's $t$-test; ${ }^{*} P<0.05,{ }^{* *} P<0.01$, ${ }^{* * *} P<0.001$; data represent mean \pm s.e.m (compared to unstimulated epithelium only) from 3 healthy donors, with 1 biological replicate per donor; $n=3$ ). c) Comparison of poly I:C induced cytokine secretion from bronchiolar and bronchial epithelial cells on-chip. Note comparable results were found for two key pro-inflammatory cytokines (unpaired Student's $t$-test; ${ }^{*} P<0.05$, ${ }^{* *} P<0.01$, ns. not significant; data represent mean \pm s.e.m (compared to unstimulated) from 3-5 different healthy donors, with 1-4 biological replicates per donor; $n=4-7$ ). 

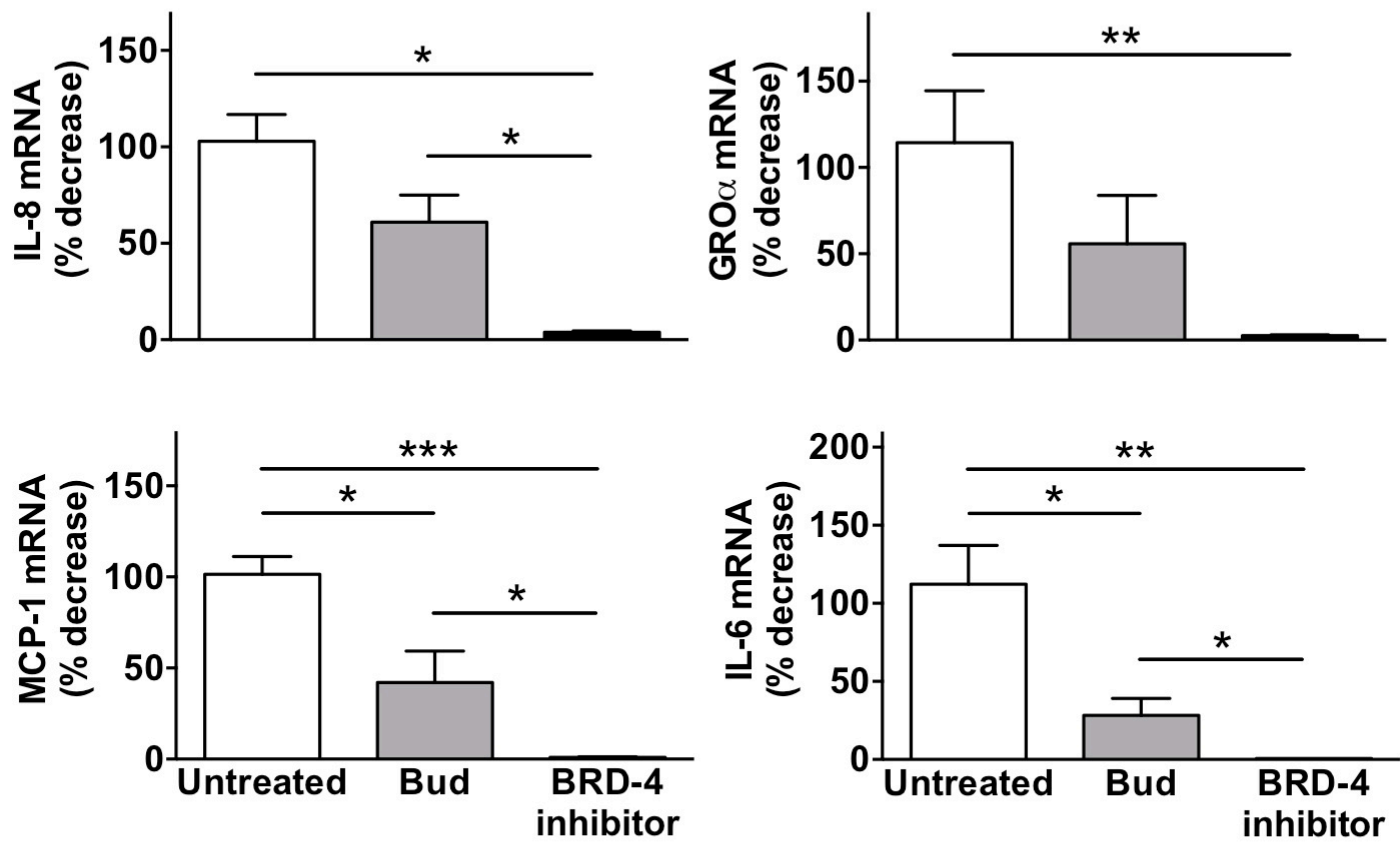

\section{Supplementary Figure 3}

Modulation of cytokine and chemokine gene expression in COPD small airway chips using a BRD-4 Inhibitor

Quantitative real-time PCR analysis of the effects of budesonide and BRD-4 inhibitor (applied as described in Fig. 5) on expression of IL-8, MCP-1, IL-6 and GROa genes in lung blood microvascular endothelial cells lysed in situ within the microvascular channel of the chips (unpaired Student's $t$-test; ${ }^{*} P<0.05$, ${ }^{* *} P<0.01$, ${ }^{* * *} P<0.001$; data represent mean \pm s.e.m (compared to untreated) from two of the donors studied in Fig. 5, with 1-2 biological replicates per condition; $n=3$ ). 